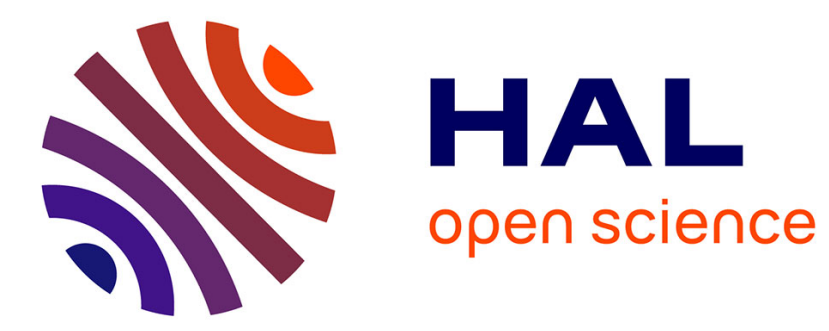

\title{
On improving matchings in trees, via bounded-length augmentations
}

\author{
Julien Bensmail, Valentin Garnero, Nicolas Nisse
}

\section{To cite this version:}

Julien Bensmail, Valentin Garnero, Nicolas Nisse. On improving matchings in trees, via boundedlength augmentations. [Research Report] Université Côte d'Azur. 2017. hal-01555465

\section{HAL Id: hal-01555465 \\ https://hal.science/hal-01555465}

Submitted on 4 Jul 2017

HAL is a multi-disciplinary open access archive for the deposit and dissemination of scientific research documents, whether they are published or not. The documents may come from teaching and research institutions in France or abroad, or from public or private research centers.
L'archive ouverte pluridisciplinaire HAL, est destinée au dépôt et à la diffusion de documents scientifiques de niveau recherche, publiés ou non, émanant des établissements d'enseignement et de recherche français ou étrangers, des laboratoires publics ou privés. 


\title{
On improving matchings in trees, via bounded-length augmentations ${ }^{1}$
}

\author{
Julien Bensmail ${ }^{\mathrm{a}}$, Valentin Garnero ${ }^{\mathrm{a}}$, Nicolas Nisse $^{\mathrm{a}}$ \\ ${ }^{a}$ Université Côte d'Azur, CNRS, Inria, I3S, France
}

\begin{abstract}
Due to a classical result of Berge, it is known that a matching of any graph can be turned into a maximum matching by repeatedly augmenting alternating paths whose ends are not covered. In a recent work, Nisse, Salch and Weber considered the influence, on this process, of augmenting paths with length at most $k$ only. Given a graph $G$, an initial matching $M \subseteq E(G)$ and an odd integer $k$, the problem is to find a longest sequence of augmenting paths of length at most $k$ that can be augmented sequentially from $M$. They proved that, when only paths of length at most $k=3$ can be augmented, computing such a longest sequence can be done in polynomial time for any graph, while the same problem for any $k \geq 5$ is NP-hard. Although the latter result remains true for bipartite graphs, the status of the complexity of the same problem for trees is not known.

This work is dedicated to the complexity of this problem for trees. On the positive side, we first show that it can be solved in polynomial time for more classes of trees, namely bounded-degree trees (via a dynamic programming approach), caterpillars and trees where the nodes with degree at least 3 are sufficiently far apart. On the negative side, we show that, when only paths of length exactly $k$ can be augmented, the problem becomes NP-hard already for $k=3$, in the class of planar bipartite graphs with maximum degree 3 and arbitrary large girth. We also show that the latter problem is NP-hard in trees when $k$ is part of the input.
\end{abstract}

Keywords: maximum matchings, bounded-length augmentations, trees.

\section{Introduction}

\subsection{Matchings and augmentations}

A matching $M$ of a (simple undirected) graph $G$ is a set of edges that are pairwise disjoint, i.e., no two edges of $M$ share an end. Matchings are rather understood objects of graph theory. A common task, for a given graph $G$, is to find a matching of $G$ that is maximum (with respect to the number of edges it includes). The maximum cardinality of a matching of $G$ is denoted by $\mu(G)$. From a well-known result of Berge [Ber57], we know that a maximum matching of $G$ can be obtained by augmenting paths arbitrarily, while it is possible.

The definition of an augmenting path is as follows. By any matching $M$ of a graph $G$, every vertex is either covered (i.e., incident to an edge in $M$ ) or exposed (i.e., not incident to any edge in $M)$. A path $P=\left(u_{1}, \cdots, u_{p}\right)$ of $G$ is said $M$-alternating if no two consecutive edges of $P$ are either both in $M$, or both not in $M$. Now, $P$ is said $M$-augmenting if it is $M$-alternating and both $u_{1}$ and $u_{p}$ are exposed (note that this implies that an augmenting path must have an odd number of edges). In the sequel, we will only call such a path augmenting, i.e., omitting $M$ when it is clear from the context. In case $P$ is augmenting, by augmenting it we mean removing from $M$ every of its edges being in $M$, and adding to $M$ all its other edges. Note that this operation, called an augmentation, results in another matching $M^{\prime}=M \Delta E(P)$ (where $\Delta$ is the symmetric difference and $E(P)$ is the set of edges of $P$ ) such that $\left|M^{\prime}\right|=|M|+1$.

Precisely, Berge's Theorem states that a matching $M$ in a graph $G$ is maximum if and only if there are no $M$-augmenting paths [Ber57]. Building on Berge's result, Edmonds, via its Blossom Algorithm [Edm65], later proved that augmenting paths in any graph $G$ can be found in polynomial time, thus that $\mu(G)$ can be computed in polynomial time. One key idea behind Berge and

${ }^{1}$ The results of this paper consist of a part of an extended abstract that have been accepted to LAGOS 2017. 
Edmonds' results is that, when converging towards a maximum matching via performing augmentations, the choice of those performed augmentations is not crucial, as they will necessarily lead to a maximum matching. This does not remain true when one is allowed to augment paths with bounded length only, as pointed out in [NSW15].

\subsection{Bounded-length augmentations}

Throughout this paper, a $(\leq k)$-augmentation is an augmentation where the augmented path has (odd) length at most $k$. For a given graph $G$ and a matching $M$ of $G$, we denote by $\mu_{\leq k}(G, M)$ the maximum size of a matching that can be reached from $M$ by performing $(\leq k)$-augmentations. These notions lead to the main problem this paper is interested in:

$(\leq k)$-Matching Problem $(M P \leq k)$

Input: A graph $G$, and a matching $M$ of $G$.

Question: What is the value of $\mu_{\leq k}(G, M)$ ?

The problem can be equivalently formulated as follows. Given a graph $G$ and a matching $M=M_{0}$ of $G$, the goal is to compute the maximum length $r$ of a sequence $\mathcal{S}=\left(P_{1}, \cdots, P_{r}\right)$ of paths, each of length at most $k$, such that: for every $1 \leq i \leq r, P_{i}$ is an $M_{i-1}$-augmenting path and $M_{i}=M_{i-1} \Delta E\left(P_{i}\right)$ (i.e., $M_{i}$ is obtained from $M_{i-1}$ by augmenting $P_{i}$ ). We say that $\mathcal{S}$ starts from $M$ and results in $M_{r}$. Note that $\left|M_{i}\right|=\left|M_{0}\right|+i$ and so $\mu_{\leq k}(G, M)=|M|+r$.

Although $\mu(G)$ can be determined in polynomial time for any graph $G$, intriguingly determining $\mu_{\leq k}(G, M)$ is an NP-hard problem in general [NSW15]. More precisely, a dichotomy result is provided concerning $M P^{\leq k}$, where the dichotomy is with respect to $k$. Namely, it is proved that $M P^{\leq 3}$ can be solved in polynomial time, while $M P^{\leq k}$ is NP-hard for every $k \geq 5$ [NSW15].

For the cases where $k \geq 5$, one may naturally wonder whether $M P^{\leq k}$ becomes polynomial-time solvable for particular classes of graphs. [NSW15] also made a first step towards that direction by showing that the NP-hardness result above holds even when the input graph is assumed to be planar, bipartite and of maximum degree 3 . One open question, though, is whether this result also extends to trees, or whether $M P^{\leq k}$ (with $k \geq 5$ ) can be solved in polynomial time for trees. This is precisely the central topic considered in this paper. Note that it is only known that $M P \leq k$ can be solved in polynomial time in the class of paths [NSW15].

Related work. The problem of finding maximum matching in bipartite graphs has been extensively studied (in particular, because it is a special case of a network flow problem). It is well known that it can be solved in polynomial-time (e.g., using the Hungarian method [Kuh55]). The first algorithm for solving the maximum matching problem in polynomial-time in general graphs is due to Edmonds [Edm65]. Then, many work has been dedicated to design more efficient algorithms [HK73, MV80, DP14]. In particular, the algorithms in [HK73, MV80] are based on augmenting paths in the non-decreasing order of their lengths. Such a method gives a good approximation since augmenting only the paths of length at most $2 k-3$ provides a $(1-1 / k)$-approximation of the maximum matching [HK73].

The problem of matching with bounded-length paths has also been studied in the context of wireless networks. in particular, it provides simple distributed algorithms to compute the scheduling of transmissions with interference [WS05, BSS09].

\subsection{Results in this paper}

In this paper, we provide both positive and negative results about the complexity of $M P^{\leq k}$ in trees. On the positive side, we show, in Section 2, that $M P^{\leq k}$ can be solved, for any odd $k \geq 5$, in polynomial time in several classes of trees. Via a dynamic programming approach, we first prove that $M P^{\leq k}$ is linear-time solvable in the class of trees with maximum degree $\Delta$. That is, $M P \leq k$ is FPT for these graphs when parameterized by $k+\Delta$. Generalizing the arguments for the path case, we then provide polynomial-time algorithms for $k$-sparse trees, i.e., trees where the nodes with degree at least 3 are at distance more than $k$, and caterpillars

Our negative results are related to the following thoughts. While trying to prove some hardness result concerning the tree instances of $M P^{\leq k}$ (for $k \geq 5$ ), we ran into the issue that allowing augmentations of length up to $k$ is a very permissive thing, which, at least in the case of trees, makes the design of a consistent NP-hardness proof not obvious. In Section 3, we thus study the behaviour 
of all those considerations in the context where only augmentations of length exactly $k$ are allowed. In other words, we consider the following problem (where the notations and terminology are derived from others above, in the obvious way):

$(=k)$-Matching Problem $\left(M P^{=k}\right)$

Input: A graph $G$, and a matching $M$ of $G$.

Question: What is the value of $\mu_{=k}(G, M)$ ?

While $M P^{\leq k}$ is NP-hard for every odd $k \geq 5$, we prove that $M P^{=k}$ is NP-hard for every odd $k \geq 3$, even when restricted to planar bipartite graphs with maximum degree 3 and arbitrarily large girth. We then focus on trees, and show that $M P^{=k}$ is NP-hard in trees when $k$ is part of the input. All our positive results also apply to $M P^{=k}$; see the concluding section for more details.

\section{Augmenting matchings via $(\leq k)$-augmentations}

In this section, we prove that, for any odd $k \geq 5, M P^{\leq k}$ can be solved in polynomial time for trees with bounded degree, $k$-sparse trees, and caterpillars. We will (sometimes implicitly) make use of the following three general statements:

Claim 2.1. When executing a sequence of augmentations, a covered vertex cannot become exposed.

Claim 2.2. After augmenting a path $P$, all vertices of $V(P)$ are covered.

Claim 2.3. Let $G$ be a graph, $M$ be a matching of $G$, and $\mathcal{S}=\left(P_{1}, \cdots, P_{r}\right)$ be a sequence of $(\leq k)$-augmentations starting from $M$. Assume $P_{i}$ and $P_{i+1}$ are disjoint for some $i<r$. Then, $\left(P_{1}, \cdots, P_{i-1}, P_{i+1}, P_{i}, P_{i+2}, \cdots, P_{r}\right)$ is a sequence of $(\leq k)$-augmentations resulting in the same matching as $\mathcal{S}$.

\subsection{Bounded-degree trees}

Using dynamic programming, we prove, in the following result, that $M P^{\leq k}$ can be solved in linear time for trees with maximum degree $\Delta$, assuming both $k$ and $\Delta$ are fixed. In other words, we show that $M P^{\leq k}$ is FPT when parametrized by $k+\Delta$.

Theorem 2.4. Let $k$ be a fixed odd integer. Let $T$ be a tree with maximum degree $\Delta$ and $M$ be a matching of $T$. Then, $\mu_{\leq k}(T, M)$ can be computed in time $O(f(k+\Delta) \cdot|V(T)|)$ for some decidable function $f$.

Proof. Let $T$ be any $n$-node tree with maximum degree $\Delta$. Let $k$ be an odd integer and let $M$ be a matching of $T$. We present an algorithm that computes $\mu_{\leq k}(T, M)$ and the corresponding sequence of paths to be augmented in time $f(\Delta, k) \cdot n$ for some computable function $f$, i.e., in linear time when both $k$ and $\Delta$ are fixed parameters. The algorithm proceeds by dynamic programming from the leaves to an arbitrary root $r \in V(T)$. For any node $v \in V(T)$, let $T_{v}$ be the subtree of $T$ rooted in $v$, let $X_{v}$ be the subtree of $T$ induced by all nodes at distance at most $k$ from $v$ and let $P_{v}$ be the set of all paths of length at most $k$ with nodes in $X_{v}$. Note that $X_{v}$ has bounded size in $\Delta$ and $k$ and so $\left|P_{v}\right|$ is $O(1)$ when $\Delta$ and $k$ are fixed parameters.

Precisely, for every node $v \in V(T)$ and for every sequence $\mathcal{S}$ of distinct paths in $P_{v}$, the algorithm computes a matching with maximum size in $T_{v}$ that can be computed from the initial matching $M$ by a sequence of augmenting paths of length at most $k$ in $T$ using the ones in $\mathcal{S}$ (respecting the order of $\mathcal{S}$ ). In other words, the algorithm computes a matching $M_{v, \mathcal{S}}$ of $T_{v}$ that is obtained from $M$ by a sequence of augmenting paths $\mathcal{S}^{\prime}$ (in $T$ ) such that $\mathcal{S}$ is a subsequence of $\mathcal{S}^{\prime}$ (i.e., all paths in $\mathcal{S}$ appear in $\mathcal{S}^{\prime}$ in the same order, not necessarily consecutively ; Note also that some paths of $\mathcal{S}$ may be subpaths of paths in $\mathcal{S}^{\prime}$ ) and that $M_{v, \mathcal{S}}$ has maximum size for these properties. By definition, the maximum solution $M_{r, \mathcal{S}}$ obtained for the root $r$ (where the maximum is taken over all possible sequences in $P_{r}$ ) corresponds to an optimal solution for $M P \leq k$.

Note that, for a given node $v \in V(T)$, the number of such partial solutions $M_{v, \mathcal{S}}$ that must be computed for $v$ is $O(1)$ when $k$ and $\Delta$ are fixed parameters. It only remains to prove that, for any node $v$ and any sequence $\mathcal{S}$, the desired solution can be computed in constant time from the solutions (the tables of the dynamic program) of the children of $v$. 
If $v$ is such that $T_{v}$ is a star (all children of $v$ are leaves) then, for every sequence $\mathcal{S}$ of paths as defined above, it is sufficient to check that the paths in $\mathcal{S}$ are compatible in $T_{v}$ with the initial matching $M$ and are pairwise compatible in $T_{v}$ (note that we a priori do not assume that the paths in $\mathcal{S}$ are augmenting paths but we have to check that it is the case at the moment when they have to be augmented). This clearly can be done in constant time since the number of these paths is $O(1)$. If the paths in $\mathcal{S}$ are compatible, then $M_{v, \mathcal{S}}$ is the matching obtained by augmenting the paths in $\mathcal{S}$. Otherwise, the returned solution is $\emptyset$ which represents the fact that augmenting the paths in $\mathcal{S}$ is not a feasible solution.

More generally, for $v \in V(T)$ and every sequence $\mathcal{S}$ of paths as defined above, the algorithm proceeds as follows. For every non-leaf child $v_{i}$ of $v$, and for every partial solution $M_{v_{i}, \mathcal{S}_{i}}$ obtained from a sequence $\mathcal{S}_{i}$ in the table of $v_{i}$, we check the compatibility of the $\mathcal{S}_{i}$ 's with $\mathcal{S}$ in $T_{v}$. The algorithm then returns a best solution $M_{v, \mathcal{S}}$ obtained from the combinations of the children's solutions. Since, they are $O(1)^{\Delta}$ such combinations, this still can be done in constant time.

\subsection{Sparse trees}

In this section, we provide a polynomial-time algorithm for solving $M P^{\leq k}$ in the cases where the graph is a $k$-sparse tree. Let us introduce a few terminology beforehand. The nodes with degree at least 3 are called $b$-nodes. A path is called a $b$-path if it contains at least one b-node, while it is called an a-path otherwise.

As we need numerous preliminary results in order to present the algorithm, let us give a first intuition of how to get a matching of size $\mu_{<k}(T, M)$ in a $k$-sparse tree $T$ starting from a matching $M$. Since the b-nodes of $T$ are far apart (i.e., a $(\leq k)$-augmenting path cannot go through two b-nodes), $T$ can be seen as a concatenation of several subdivided stars $T_{1}, \cdots, T_{q}$ glued along some of their branches. Hence, a sequence of $(\leq k)$-augmentations in $T$ is made up of subsequences of $(\leq k)$-augmentations in the $S_{i}$ 's, that eventually have to get combined somehow. One of the main goals throughout this section is thus to comprehend how to get, via $(\leq k)$-augmentations, a matching of size $\mu_{\leq k}(S, M)$ for any subdivided star $S$ and initial matching $M$.

A subdivided star, generally speaking, can be seen as a combination of paths attached at one node. Solving $M P^{\leq k}$ in a path can be done easily, as first shown by Nisse, Salch and Weber [NSW15], as it suffices to go from left to right and augment paths of close exposed nodes as they are encountered. In this section, we prove that, in a subdivided star $S$, a matching of size $\mu_{\leq k}(S, M)$ can be attained, roughly speaking, by performing at most one $(\leq k)$-augmentation through the b-node, and several $(\leq k)$-augmentations along the branches (i.e., apply the path algorithm onto a forest of paths). In particular, the augmentation through the b-node is proved to be necessary in a very particular context only.

We now start off by proving several lemmas. In what follows, given two paths $Q$ and $P$, we note $Q \cap P$ for $V(P) \cap V(Q)$, i.e., we consider vertex-intersections.

Lemma 2.5. Let $T$ be a $k$-sparse tree, $M$ be a matching, and $\mathcal{S}=\left(P_{1}, \cdots, P_{r}\right)$ be a sequence of $(\leq k)$-augmentations starting from $M$. Then, two b-paths in $\mathcal{S}$ intersect only if they contain the same b-node.

Proof. Let $1 \leq i<j \leq r$, and let $P_{i}$ and $P_{j}$ be two b-paths containing the b-nodes $u$ and $v$, respectively. Because $T$ is $k$-sparse, no path in $\mathcal{S}$ can contain more than one b-node. For purpose of contradiction, let us assume that $u \neq v$ and $P_{i} \cap P_{j} \neq \emptyset$. Since $P_{i} \cap P_{j} \neq \emptyset$, there cannot be any b-node on the path from $u$ and $v$ since, otherwise, $u$ and $v$ would be at distance at least $2 k+2$ from each other and $P_{i}$ and $P_{j}$ (of length at most $k$ each) could not intersect. Hence, $P_{i}$ and $P_{j}$ must intersect on the path $Q$ between $u$ and $v$, that consists only of nodes of degree 2 . In particular, there must be an end $x$ of $P_{j}$ that is in $Q \cap P_{i}$. On the one end, $x$ must be exposed since $P_{j}$ is an augmenting path. On the other hand, after $P_{i}$ has been augmented, $x$ is covered. A contradiction.

The following lemmas are devoted to prove that, in sparse trees, augmentation sequences can be restricted to have some specific structure. First, we show that any sequence of augmentations can be transformed into an equivalent sequence that first augments b-paths, and then a-paths. 
Lemma 2.6. Let $T$ be a $k$-sparse tree, $M$ be a matching, and $\mathcal{S}=\left(P_{1}, \cdots, P_{r}\right)$ be a sequence of $(\leq k)$-augmentations starting from $M$. Let $1 \leq i<r$ be such that $P_{i}$ is an a-path and $P_{i+1}$ is a b-path. Then, there exist two a-paths $A$ and $B$ such that $A \cap B=\emptyset$, at most one of them, say $A$, is a b-path and $\mathcal{S}^{\prime}=\left(P_{1}, \cdots, P_{i-1}, A, B, P_{i+2}, \cdots, P_{r}\right)$ is a sequence of $(\leq k)$-augmentations resulting in the same matching as $\mathcal{S}$.

Proof. By Claim 2.3, the lemma holds if $P_{i} \cap P_{i+1}=\emptyset$. Let us assume that $P_{i} \cap P_{i+1} \neq \emptyset$. If $P_{i}$ is included in $P_{i+1}$, then we can augment the two paths of $P_{i} \Delta P_{i+1}$ (where $\Delta$ is the symmetric difference) instead of $P_{i}$ and $P_{i+1}$. Clearly at most one of these paths is a b-path and it can be augmented first. Otherwise (if $P_{i}$ not included in $P_{i+1}$ ) then let $x$ be the end of $P_{i+1}$ belonging to $V\left(P_{i}\right)$ (this $x$ must exist since $P_{i}$ is an a-path not included in $P_{i+1}$ ). Then $x$ must be covered after the augmentation of $P_{i}$, contradicting the fact that $P_{i+1}$ is augmenting.

Note that repeating the argument of previous lemma, we can ensure that there exists an optimal sequence of augmentations that augments the b-paths first. We now show that any sequence of augmentations can be transformed into an equivalent sequence where all a-paths are vertex-disjoint.

Lemma 2.7. Let $T$ be a tree, $M$ be a matching, and $\mathcal{S}=\left(P_{1}, \cdots, P_{r}\right)$ be a sequence of $(\leq k)$ augmentations starting from $M$. Let $1 \leq i<r$ be such that $P_{i}$ and $P_{i+1}$ are a-paths. Then, there exist two a-paths $A$ and $B$ such that $A \cap B=\emptyset$ and $\mathcal{S}^{\prime}=\left(P_{1}, \cdots, P_{i-1}, A, B, P_{i+2}, \cdots, P_{r}\right)$ is a sequence of $(\leq k)$-augmentations resulting in the same matching as $\mathcal{S}$.

Proof. If $P_{i} \cap P_{i+1}=\emptyset$, then $A=P_{i}$ and $B=P_{i+1}$ satisfy the lemma. Hence, let us assume that $P_{i} \cap P_{i+1} \neq \emptyset$. Let $P_{i}=\left(v_{a}, \cdots, v_{b}\right)$ and $P_{i+1}=\left(v_{1}, \cdots, v_{c}\right)$. After augmenting $P_{i}$, the nodes $v_{a}$ and $v_{b}$ are covered, and before augmenting $P_{i+1}$, the nodes $v_{1}$ and $v_{c}$ were exposed. Now, since $P_{i}$ and $P_{i+1}$ are a-paths, it implies that $V\left(P_{i}\right) \subset V\left(P_{i+1}\right)$ and, thus, $P_{i+1}=\left(v_{1}, \cdots, v_{a}, \cdots, v_{b}, \cdots, v_{c}\right)$. Then, setting $A=\left(v_{1}, \cdots, v_{a}\right)$ and $B=\left(v_{b}, \cdots, v_{c}\right)$ proves the lemma.

From previous lemmas and claims, we get:

Corollary 2.8. Let $T$ be a $k$-sparse tree with b-nodes $\left\{v_{1}, \cdots, v_{o}\right\}, M$ be a matching, and $\mathcal{S}=$ $\left(P_{1}, \cdots, P_{r}\right)$ be a sequence of $(\leq k)$-augmentations starting from $M$. Then, there is a sequence $\mathcal{S}^{\prime}$ of $(\leq k)$-augmentations starting from $M$, resulting in the same matching as $\mathcal{S}$, that consists of, in order:

- a sequence of b-paths each containing one of the $v_{i}$ 's, such that two b-paths containing different $v_{i}$ 's do not intersect, and moreover the b-paths containing a same $v_{i}$ are consecutive, and

- a sequence of a-paths that are pairwise vertex-disjoint and vertex-disjoint from the previous augmented b-paths.

A sequence satisfying the properties of Corollary 2.8 is called partially-well structured. Next, we characterize the "structure" of the b-paths augmented in a sparse tree. First, let us consider the case when a b-node is not initially covered by the matching.

Lemma 2.9. Let $T$ be a $k$-sparse tree, $M$ be a matching, and $\mathcal{S}=\left(P_{1}, \cdots, P_{r}\right)$ be a sequence of $(\leq k)$-augmentations starting from $M$. Let $v$ be any b-node of $T$. If $v$ is exposed by $M$, then there exists a partially-well structured sequence $\mathcal{S}^{\prime}$ of $(\leq k)$-augmentations starting from $M$, resulting in the same matching as $\mathcal{S}$, and such that at most one path of $\mathcal{S}^{\prime}$ contains $v$. Moreover, this sequence can be obtained by modifying only the b-paths of $\mathcal{S}$ that contain $v$.

Proof. By Corollary 2.8, we may assume that $\mathcal{S}$ is partially-well structured. If at most one path of $\mathcal{S}$ contains $v$, then we are done. Otherwise, let $P$ and $Q$ be the first two paths of $\mathcal{S}$ that contain $v$. Since $\mathcal{S}$ is partially-well structured, $P$ and $Q$ are consecutive in $\mathcal{S}$, let $P=P_{i}$ and $Q=P_{i+1}$ for some $i<r$. Since $v$ is exposed by $M$, necessarily $P$ must be a path that ends in $v$ and, because $T$ is $k$ sparse and $P$ has length at most $k$, all nodes of $P$ (but $v$ ) have degree at most 2 in $T$. Let us set $P=$ $\left(v_{1}, \cdots, v_{j}=v\right)$. After having augmented $P$, the edge $v_{j-1} v_{j}$ belongs to the matching and all nodes of $P$ are covered. Therefore, the only way for $Q$ to be an augmenting path containing $v$ is to fully contain $P$. So $Q=\left(w_{1}, \cdots, w_{s}, v_{1}, \cdots, v_{j}=v, u_{1}, \cdots, u_{h}\right)$ where all nodes of $Q$ but $v$ have degree at most 2 . Hence, the sequence $\mathcal{S}^{\prime}=\left(P_{1}, \cdots, P_{i-1},\left(w_{1}, \cdots, w_{s}, v_{1}\right),\left(v, u_{1}, \cdots, u_{h}\right), P_{i+2}, \cdots, P_{r}\right)$ 
is a sequence of $(\leq k)$-augmenting paths resulting in the same matching as $\mathcal{S}$ and containing strictly less paths containing $v$. Reordering $\mathcal{S}^{\prime}$ using Lemma 2.6, we obtain a partially-well structured sequence containing the same paths as in $\mathcal{S}^{\prime}$. Repeating this process until at most one path contains $v$ allows us to obtain a sequence satisfying the lemma.

Now, we characterize the "structure" of the b-paths containing a b-node that is covered by the initial matching. Let $T$ be a $k$-sparse tree with an initial matching $M$ and let $v$ be a b-node that is covered by $M$. Let $\mathcal{S}=\left(P_{1}, \cdots, P_{r}\right)$ be a sequence of $(\leq k)$-augmentations all of which pass through $v$. The path $P_{1}$ must go from $T_{i_{1}}$, the component of $T-\{v\}$ that contains the node matched with $v$ by $M$, to some other component $T_{i_{2}}$ of $T-\{v\}$. After having augmented $P_{1}$, the node matched with $v$ must be in $T_{i_{2}}$. Then, $P_{2}$ must go from $T_{i_{2}}$ to another component $T_{i_{3}}$ of $T-\{v\}$, i.e., $i_{3} \neq i_{2}$ but $i_{3}$ may be equal to $i_{1}$. Going on that way, $\mathcal{S}$ is fully characterized by the sequence $\left(T_{i_{1}}, \cdots, T_{i_{r+1}}\right)$ of components of $T-\{v\}$ such that, for every $1 \leq j \leq r$, we have $i_{j} \neq i_{j+1}$. Precisely, for any $j \leq r$, after having augmented $P_{1}, \cdots, P_{j}$, the path $P_{i}$ goes from the exposed node of $T_{i_{j}}$ that is the closest to $v$, to the exposed node of $T_{j+1}$ that is the closest to $v$. We say that such a sequence starts in $T_{i_{1}}$ and finishes in $T_{i_{r+1}}$. Let us call the sequence $\mathcal{S}$ to be unlooping if the indices in $\left\{i_{1}, \cdots, i_{r+1}\right\}$ are pairwise distinct. Note that, in particular, the length of such a sequence is bounded by the degree of $v$ minus 1 .

Lemma 2.10. Let $T$ be a $k$-sparse tree, $M$ be a matching, and $\mathcal{S}=\left(P_{1}, \cdots, P_{r}\right)$ be a sequence of $(\leq k)$-augmentations starting from $M$. Let $v$ be any $b$-node of $T$. If $v$ is covered by $M$, then there exists a partially-well structured sequence $\mathcal{S}^{\prime}$ of $(\leq k)$-augmentations starting from $M$, resulting in the same matching as $\mathcal{S}$, and such that the subsequence of b-paths containing $v$ is unlooping. Moreover, this sequence can be obtained by modifying only the b-paths of $\mathcal{S}$ that contain $v$.

Proof. By Corollary 2.8, we may assume that $\mathcal{S}$ is partially-well structured. Let $\mathcal{S}^{\prime}=\left(P_{1}^{\prime}, \cdots, P_{s}^{\prime}\right)$ be the subsequence of (consecutive) b-paths of $\mathcal{S}$ that contain $v$. If $\mathcal{S}^{\prime}$ is unlooping, then we are done. Otherwise, let $\left(T_{i_{1}}, \cdots, T_{i_{s+1}}\right)$ be the sequence of components of $T-\{v\}$ that characterizes $\mathcal{S}^{\prime}$ (as in the paragraph above). Let $y<x \leq s$ be such that the indices in $\left\{i_{y}, \cdots, i_{x}\right\}$ are pairwise distinct and $i_{y}=i_{x+1}$. We show that, in $\mathcal{S}^{\prime}$ (and so in $\mathcal{S}$ ), we can replace the paths $P_{y}, \cdots, P_{x}$ by as many a-paths (i.e., not passing through $v$ ). For every $y \leq j \leq x$, let $d_{j}$ be the end of $P_{j}$ in the component $T_{i_{j}}$ and let $f_{j}$ be its end in the component $T_{i_{j+1}}$. For every $y \leq j<x$, let $Q_{j}$ be the path between $f_{j}$ and $d_{j+1}$, and let $Q_{x}$ be the path between $d_{y}$ and $f_{x}$. Then, the sequence $\mathcal{S}^{\prime \prime}=\left(P_{1}^{\prime}, \cdots, P_{y-1}^{\prime}, Q_{y}, \cdots, Q_{x}, P_{x+1}^{\prime}, \cdots, P_{s}^{\prime}\right)$ results in the same matching as $\mathcal{S}^{\prime}$. Moreover, the paths $Q_{y}, \cdots, Q_{x}$ are $a$-paths, and according to Lemma 2.6, they can be re-ordered to obtain a partially-well structured sequence. In this latter sequence, the number of times that the b-paths containing $v$ are "looping around $v$ " has been reduced by 1 . Hence, repeating this process leads to the desired sequence of $(\leq k)$-augmentations.

In what follows, we deal with sequences of augmentations having a particular shape. Let $T$ be a $k$-sparse tree and $M$ be a matching. Denote by $K=\left\{c_{1}, \cdots, c_{p}\right\}$ and $U=\left\{u_{1}, \cdots, u_{q}\right\}$ the sets of b-nodes respectively covered and exposed by $M$. A sequence $\mathcal{S}$ of $(\leq k)$-augmentations starting from $M$ is said well structured if it consists of, in order:

- a sequence of b-paths containing the $u_{i}$ 's, where each $u_{i}$ is contained in at most one b-path,

- for every $i \leq p$, there is one unlooping (possibly empty) sequence of b-paths containing $c_{i}$ (in particular, the b-paths containing $c_{i}$ are consecutive), and

- a sequence of $a$-paths.

Moreover, every two paths of the whole sequence intersect if and only if they contain the same b-node. Clearly, a well structured sequence is also partially-well structured.

All results proved so far allow to easily derive the following theorem.

Theorem 2.11. Let $T$ be a $k$-sparse tree, $M$ be a matching, and $\mathcal{S}$ be a sequence of $(\leq k)$ augmentations starting from $M$. Then, there exists a well-structured sequence of $(\leq k)$-augmentations starting from $M$ and resulting in the same matching as $\mathcal{S}$. 
Lemma 2.12. Let $T$ be a $k$-sparse tree, $M$ be a matching, and $\mathcal{S}$ be a sequence of $(\leq k)$ augmentations starting from $M$ and resulting in a matching $M^{\prime}$. Let $v$ be any node that is contained in a path of $\mathcal{S}$. Then, there exists a sequence of $(\leq k)$-augmentations starting from $M$ such that none of its paths contains $v$, and resulting in a matching $M^{\prime \prime}$ of size at least $\left|M^{\prime}\right|-1$. Moreover, if $v$ is a b-node, then $M$ and $M^{\prime \prime}$ only differ in some edges of the paths of $\mathcal{S}$ containing $v$.

Proof. By Theorem 2.11, we may assume that $\mathcal{S}$ is well-structured.

- Let us first assume that $v$ is a b-node. If at most one path of $\mathcal{S}$ contains $v$, then removing it (if it exists) leads to the desired result. Hence, we may assume that there is a unique subsequence $\mathcal{S}^{\prime}=\left(P_{1}, \cdots, P_{r}\right)$ of b-paths of $\mathcal{S}$ containing $v$. Moreover, this subsequence is unlooping. Let $\left(T_{1}, \cdots, T_{r+1}\right)$ be the sequence of components of $T-\{v\}$ that characterizes $\mathcal{S}^{\prime}$. For every $1 \leq i \leq r$, let $d_{i}$ be the end of $P_{i}$ in the component $T_{i}$ and let $f_{i}$ be its end in the component $T_{i+1}$. For every $1 \leq i<r$, let $Q_{i}$ denote the path between $f_{i}$ and $d_{i+1}$. Then, replacing the sequence $\mathcal{S}^{\prime}$ by $\left(Q_{1}, \cdots, Q_{r-1}\right)$ in $\mathcal{S}$ results in a matching of size $\left|M^{\prime}\right|-1$ and that differs from $M$ only on the path between $d_{1}$ and $f_{r}$. Moreover, no paths of the resulting sequence contain $v$.

- If $v$ is not a b-node, then we consider two cases. If $v$ is contained in an a-path of $\mathcal{S}$, then it is sufficient to remove this path from $\mathcal{S}$ (because it is disjoint from any other path, since $\mathcal{S}$ is wellstructured). Otherwise, $v$ belongs to one path of an unlooping sequence $\mathcal{S}^{\prime}=\left(P_{1}, \cdots, P_{r}\right)$. For every $1 \leq i \leq r$, let $d_{i}$ be the starting node of $P_{i}$ and let $f_{i}$ be its end. Let $j \leq r$ be the first index such that $v \in V\left(P_{j}\right)$. Then, it is sufficient to replace $\mathcal{S}^{\prime}$ by $\left(P_{1}, \cdots, P_{j-1}, P_{j+1}^{\prime}, \cdots, P_{r}^{\prime}\right)$ where, for every $j<i \leq r$, we denote by $P_{i}^{\prime}$ the path between $d_{i}$ and $f_{i-1}$.

Lemma 2.13. Let $T$ be a $k$-sparse tree, $M$ be a matching, and $v$ be a b-node covered by $M$. Let $\mathcal{B}=\left\{T_{1}, \cdots, T_{r}\right\}$ be any subset of at least two components of $T-\{v\}$. It can be decided in polynomial time (in $V(T)$ ) whether it exists an unlooping sequence of $(\leq k)$-augmentations intersecting $v$, starting in $T_{1}$, finishing in $T_{r}$ and only passing through components of $\mathcal{B}$.

Proof. A trivial necessary condition is that $v$ is matched by $M$ with a node in $T_{1}$ (otherwise no sequence of augmenting paths containing $v$ can start in $T_{1}$ ). Hence, we may assume that it is the case.

Consider the following auxiliary digraph with vertex-set $\left\{v_{1}, \cdots, v_{r}\right\}$. For every $2 \leq j \leq r$, add an arc from $v_{1}$ to $v_{j}$ if the first exposed node of $T_{1}$ (the one that is closest to $v$ ) is at distance at most $k$ from the first exposed node of $T_{j}$ (in which case, the path between these two nodes is augmenting and has length at most $k$ ). Then, for every $2 \leq i<j \leq r$, add an arc from $v_{i}$ to $v_{j}$ if the second exposed node of $T_{i}$ is at distance at most $k$ from the first exposed node of $T_{j}$. It is easy to show that a desired sequence exists if and only if there is a directed (simple) path from $v_{1}$ to $v_{r}$ in that digraph, which can be checked by a BFS algorithm.

Before going on, we present the following lemma that leads to a much simpler proof than that in [NSW15] of the linear-time algorithm to compute $\mu_{\leq k}$ in paths. Let $P$ be a path graph and $M$ be a matching. Let $v_{1}, \cdots, v_{r}$ be the exposed nodes in order, say, from "left to right". First, as in [NSW15], if two consecutive exposed nodes (i.e., $v_{i}$ and $v_{i+1}$ for some $i<r$ ) are at distance strictly more than $k$, then all edges between them can be removed. When this process ends, we are left with a set of disjoint paths (subpaths of $P$ ), whose every two consecutive exposed nodes are at distance at most $k$. The following lemma expresses a simple algorithm to deal with this kind of instances.

Lemma 2.14. Let $P$ be a path, and $M$ be matching such that two consecutive exposed nodes are at distance at most $k$. Let $v_{1}, \cdots, v_{r}$ denote the exposed nodes in order, say, from "left to right". Then, the sequence of paths $\left(P_{i}\right)_{1<i<\mid r / 2\rfloor}$, where $P_{i}$ denotes the path going from $v_{2 i-1}$ to $v_{2 i}$, is a sequence of $(\leq k)$-augmentations starting from $M$ and resulting in a matching of size $\mu_{\leq k}(P, M)$.

Proof. We only need to show that the sequence is optimal. Each time a path is augmented in $P$, two exposed nodes become covered. Therefore, any sequence of augmentations contains at most $\lfloor r / 2\rfloor$ paths. Since the size of the final matching is $|M|$ plus the number of augmented paths, the proposed sequence is optimal. 
In the context of sparse trees, the previous proof motivates us to consider the parity of the number of exposed nodes in the "branches". Let $T$ be a tree rooted in some node $r$. A b-node of $T$ is called a lowest $b$-node if it has no other b-nodes as descendant. Any component of $T-\{v\}$ that consists of descendants of $v$ is called a child-branch at $v$. The component of $T-\{v\}$ that contains the parent of $v$ is called the parent-branch at $v$. For a matching $M$ of $T$, a child-branch $B$ at $v$ is called even (resp., odd) if the number of exposed nodes in $B$ is even (resp., odd). Finally, the pair $(T, M)$ is said clean if there are no two exposed nodes at distance strictly more than $k$ such that all nodes on the path between them are covered and have degree 2.

Lemma 2.15. Let $T$ be a $k$-sparse tree, $M$ be a matching such that $(T, M)$ is clean, and $\mathcal{S}$ be a well-structured sequence of $(\leq k)$-augmentations starting from $M$ and resulting in a matching $M^{\prime}$. Let $v$ be any lowest b-node. Let $\mathcal{S}^{\prime}$ be the subsequence of paths of $\mathcal{S}$ containing $v$. If $\mathcal{S}^{\prime}$ starts or ends in an even child-branch at $v$, then there exists a sequence of $(\leq k)$-augmentations starting from $M$ such that none of its paths contains $v$, and resulting in a matching $M^{\prime \prime}$ of size at least $\left|M^{\prime}\right|$.

Proof. Let $\mathcal{S}$ be a well-structured optimal sequence for $(T, M)$ having the properties described in Theorem 2.11. If no path of $\mathcal{S}$ contains both $v$ and a node in some even child-branch, then the result clearly holds.

Now assume that at least one path intersects $v$. Since $\mathcal{S}$ is well-structured, there is a subsequence $\mathcal{S}^{\prime}=\left(P_{1}, \cdots, P_{r}\right)$ of b-paths of $\mathcal{S}$ containing $v$ (possibly $r=1$ ). Moreover, this subsequence is unlooping. Let $\left(T_{1}, \cdots, T_{r+1}\right)$ be the sequence of components of $T-\{v\}$ that characterizes $\mathcal{S}^{\prime}$. Moreover, either $T_{1}$ or $T_{r+1}$ is an even child-branch. Let us assume it is $T_{1}$, the other case being symmetric, and let $v_{1}, \cdots, v_{2 h}$ be the exposed nodes (by $M$ ) on $T_{1}$, with $v_{1}$ being the closest to $v$. For every $1 \leq i \leq r$, let $d_{i}$ be the end of $P_{i}$ in the component $T_{i}$ and let $f_{i}$ be its end in the component $T_{i+1}$. For every $1 \leq i<r$, let $Q_{i}$ denote the path (of $T_{i+1}$ ) between $f_{i}$ and $d_{i+1}$. Replacing, in $\mathcal{S}$, the paths $\left(P_{1}, \cdots, P_{r}\right)$ and all paths of $\mathcal{S}$ strictly included in $T_{1}$, by the paths $\left(Q_{1}, \cdots, Q_{r-1}\right)$ and by the paths from $v_{2 i-1}$ to $v_{2 i}$, for every $1 \leq i \leq h$, we obtain a sequence of $(\leq k)$-augmentations none of which contains $v$, and resulting in a matching of same size as $M$. Hence, we are back to the first case.

Lemma 2.16. Let $T$ be a rooted $k$-sparse tree, $M$ be a matching such that $(T, M)$ is clean, and $\mathcal{S}$ be an optimal well-structured sequence of $(\leq k)$-augmentations starting from $M$. Let $v$ be any lowest b-node with child-branches $B_{1}, \cdots, B_{h}$. For any child-branch $B_{i}$ at $v$, let $x_{i}$ be its number of exposed nodes and let $\delta_{i}=1$ if $B_{i}$ is an even branch and $\delta_{i}=0$ otherwise. Assume that $\mathcal{S}$ contains an unlooping sequence $\left(P_{1}, \cdots, P_{s}\right)$ of paths containing $v$, characterized by a sequence $\mathcal{B}$ of components of $T-\{v\}$. Let $Z$ be the number of paths augmented by $\mathcal{S}$ that intersect the subtree $T_{v}$ of $T$ rooted at $v$. Then:

1. If $\mathcal{B}$ contains only child-branches at $v$, w.l.o.g., $\mathcal{B}=\left(B_{1}, \cdots, B_{s+1}\right)$, then, $Z=1-\delta_{1}-$ $\delta_{s+1}+\sum_{1 \leq i \leq h}\left\lfloor\left(x_{i}\right) / 2\right\rfloor$.

2. If $\mathcal{B}$ contains (but neither starts nor ends by) the parent-branch at $v$, w.l.o.g., $\mathcal{B}=\left(B_{1}, \cdots, B_{s+1}\right)$ and the parent-branch is $B_{q}(1<q<s+1)$, then $Z=2-\delta_{1}-\delta_{s+1}+\sum_{1 \leq i \leq h, i \neq q}\left\lfloor\left(x_{i}\right) / 2\right\rfloor$.

3. If $\mathcal{B}$ starts or finishes by the parent-branch at $v$, w.l.o.g., $\mathcal{B}=\left(B_{1}, \cdots, B_{s+1}\right)$ and the parentbranch is $B_{1}$, then $Z=1-\delta_{s+1}+\sum_{1<i \leq h}\left\lfloor\left(x_{i}\right) / 2\right\rfloor$.

In particular, $Z$ depends only on the parity of the first and final child-branches. Furthermore, if no paths of $\mathcal{S}$ contain $v$, then $Z=\sum_{1 \leq i \leq h}\left\lfloor\left(x_{i}\right) / 2\right\rfloor$.

Proof. We prove the result for the first item, the second and third items being similar. The number of paths augmented in $T_{v}$ by $\mathcal{S}$ is

$$
s+\left\lfloor\left(x_{1}-1\right) / 2\right\rfloor+\left\lfloor\left(x_{s+1}-1\right) / 2\right\rfloor+\sum_{1<i<s+1}\left\lfloor\left(x_{i}-2\right) / 2\right\rfloor+\sum_{s+2 \leq i \leq h}\left\lfloor x_{h} / 2\right\rfloor .
$$

Indeed, augmenting the $s$ paths $P_{1}, \cdots, P_{s}$ "consumes" one exposed node in both $B_{1}$ and $B_{s+1}$ and two exposed nodes in every branch $B_{2}, \cdots, B_{s}$. Furthermore, since $\mathcal{S}$ is an optimal well-structured sequence, and according to Lemma 2.14, the number of augmented paths in each branch is the number of remaining exposed nodes divided by 2 (floor). Reorganizing the sum gives the result.

The last sentence of the statement is trivial, and follows e.g., from Lemma 2.14. 


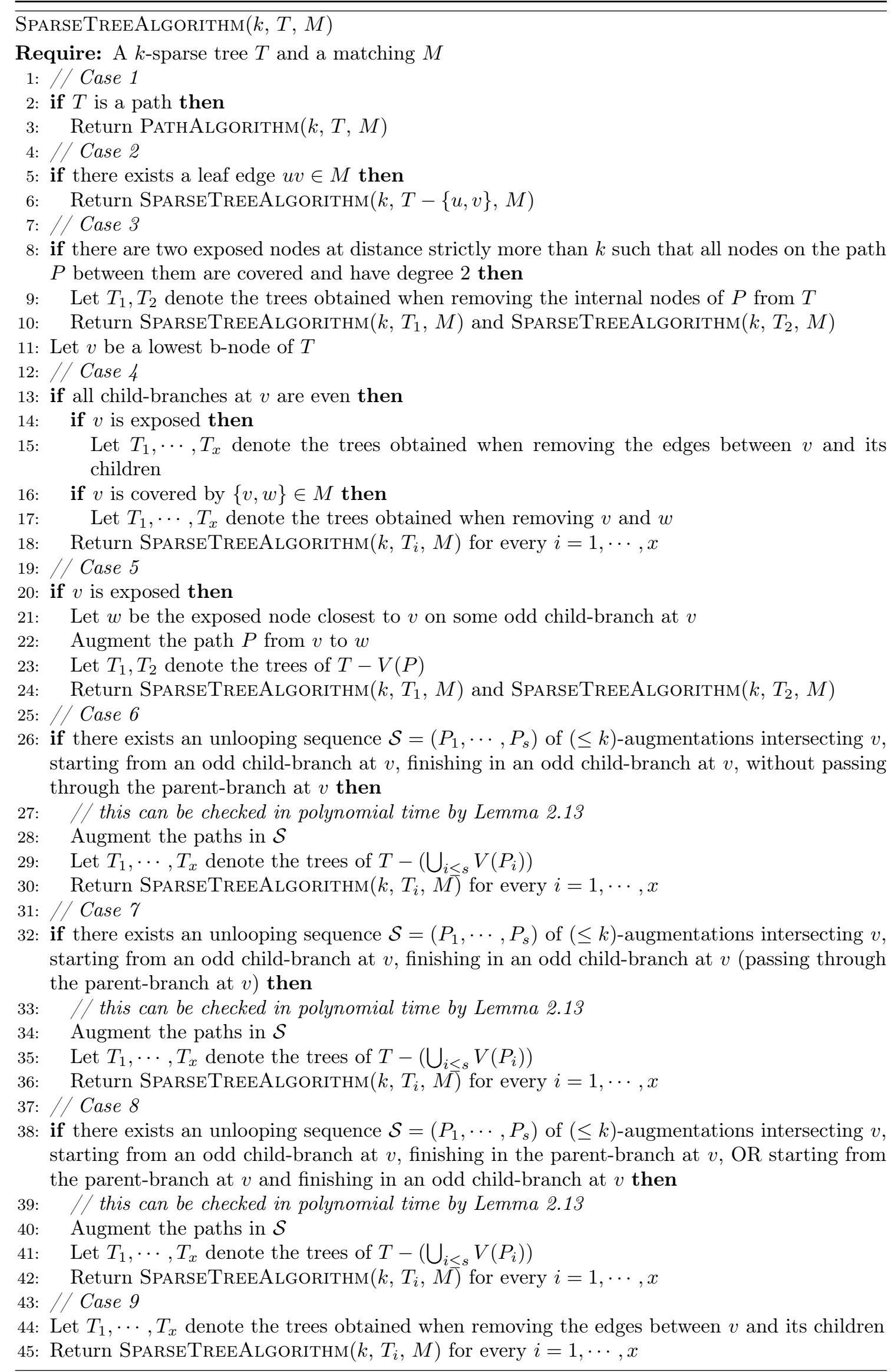


We are now ready to introduce a polynomial-time algorithm for solving $M P^{\leq k}$ in the case of $k$-sparse trees. For purpose of simplification, the algorithm we introduce only computes a matching of size $\mu_{\leq k}(G, M)$. However, it can be modified easily, by memorizing the paths that have been augmented, so that it also provides a corresponding sequence of $(\leq k)$-augmentations.

Theorem 2.17. For any $k$-sparse tree $T$ and matching $M$, SparseTreeAlgorithm $(k, T, M)$ computes in polynomial time (in $|V(T)|$ ) a matching of size $\mu_{\leq k}(T, M)$ by performing $(\leq k)$ augmentations starting from $M$.

Proof. The algorithm PathAlgorithm mentioned in Case 1 is the linear-time algorithm for solving $M P^{\leq k}$ in the case of paths mentioned in Lemma 2.14. SPARSETREeAlgorithm is a recursive algorithm. The fact that it runs under polynomial time is obvious since all tests can indeed be performed in polynomial time (in Cases 6, 7 and 8, this is true by Lemma 2.13), and, in any of the cases, the algorithm is recursively applied on vertex-disjoint (strictly smaller) subtrees of $T$.

Let us prove the correctness of SPARSETREEALGORITHM, i.e., the fact that we eventually obtain a matching of size $\mu_{\leq k}(T, M)$ from $M$, by induction on the size of $T$. The result clearly holds in Cases 1,2,3. In all remaining cases, the proof follows the same scheme: we start from a well-structured sequence $\mathcal{S}$ of $(\leq k)$-augmentations starting from $M$ and resulting in a matching of size $\mu_{\leq k}(T, M)$ (it exists by Theorem 2.11), and we show that the algorithm achieves a matching of (at least) the same size.

Case 4. In that case, $v$ has only even child-branches. We prove the correctness when $v$ is exposed, the case when $v$ is covered being similar. We prove that there is a sequence of $(\leq k)$ augmentations starting from $M$ and resulting in a matching of size $\mu_{\leq k}(T, M)$ that can be obtained by considering independently the child-branches $B_{1}, \cdots, B_{s}$ at $v$ and $T-\left(\bigcup_{i \leq s} V\left(B_{i}\right)\right)$. Since it is what is done by SpArseTreeAlgorithm, the result holds by induction.

If $\mathcal{S}$ does not contain any path containing $v$ and some node of a child-branch, then the result clearly holds. Otherwise, since all child-branches at $v$ are even, by Lemma 2.15 there exists an optimal sequence not intersecting $v$.

Case 5. In that case, $v$ is exposed and has at least one odd child-branch. Since $\mathcal{S}$ is well-structured, there is at most one path $P$ of it that contains (and ends in) $v$. Let $B$ be any odd child-branch at $v$, and let $v_{1}, \cdots, v_{2 h+1}$ be the exposed nodes (by $M$ ) on $B$, with $v_{1}$ being the closest to $v$. Because $\mathcal{S}$ is well-structured, by Lemma 2.14 there are, in $\mathcal{S}$, at most $h$ augmenting paths strictly included in $B$. Set $v=v_{0}$. We simply replace $P$ and these paths in $B$ by the paths $P_{i}$ from $v_{2 i}$ to $v_{2 i+1}$ for every $0 \leq i \leq h$. The obtained sequence can clearly be obtained by first augmenting $P_{0}$ and then considering all components of $T-P_{0}$ independently. This is what SparseTreeAlgorithm does. Hence, the result holds by induction.

Case 6. In that case, there is an unlooping sequence $\mathcal{S}^{\prime}=\left(P_{1}, \cdots, P_{s}\right)$ of paths containing $v$, characterized by a sequence $\left(T_{1}, \cdots, T_{s+1}\right)$ of components of $T-\{v\}$. Moreover, $T_{1}$ and $T_{s+1}$ are odd child-branches at $v$, and $T_{2}, \cdots, T_{s}$ are even child-branches at $v$.

In that case, SparseTreeAlgorithm first augments the paths in $\mathcal{S}^{\prime}$ and then in the components of $T-\bigcup_{i \leq s} V\left(P_{i}\right)$ independently. Let $B_{1}, \cdots, B_{h}$ be the child-branches at $v$ and, for every $i \leq h$, let $x_{i}$ be the number of exposed nodes of $B_{i}$. Let $Q$ be the parent-branch at $v$. Said differently, SpArSeTreeAlgorithm computes an optimal solution for $Q$ (by induction) and, by Lemma 2.16, augments $1+\sum_{i \leq h}\left\lfloor x_{i} / 2\right\rfloor$ paths in $T_{v}$. Let us compare this number of augmentations with the one obtained by the optimal sequence $\mathcal{S}$. There are several cases to consider.

- No path of $\mathcal{S}$ contains $v$ and some node of a child-branch at $v$. Then $\mathcal{S}$ deals with $Q \cup\{v\}$ and $T_{v}-\{v\}$ independently. By Lemma 2.16, at most $\sum_{i \leq h}\left\lfloor x_{i} / 2\right\rfloor$ paths may be augmented in $T_{v}$. Moreover, by Lemma 2.12, in $Q \cup\{v\}$, the number of paths that may be augmented is at most the maximum number of paths that may be augmented in $Q$ plus 1 . Overall, $\mathcal{S}$ augments at most the same number of paths as SPARSETREEALGORITHM.

- No path of $\mathcal{S}$ contains $v$ and some node of the parent-branch at $v$. Then $\mathcal{S}$ deals with $Q$ and $T_{v}$ independently (as SparseTreeAlgorithm). By Lemma 2.16, at most $1+$ 
$\sum_{i \leq h}\left\lfloor x_{i} / 2\right\rfloor$ paths may be augmented. So $\mathcal{S}$ does the same as SparseTreeAlgorithm (possibly augmenting a different unlooping sequence around $v$ ).

- $\mathcal{S}$ contains an unlooping sequence around $v$ that starts or finishes in the parent-branch at $v$. Then, by Lemma $2.16, \mathcal{S}$ augments at most $1+\sum_{1<i<h}\left\lfloor\left(x_{i}\right) / 2\right\rfloor$ paths intersecting $T_{v}$ and then computes an optimal solution in a subtree of $\bar{Q}$ (precisely an optimal solution of $Q$ minus the nodes of the paths of the unlooping sequence). Therefore, the solution computed by SparseTreeAlgorithm has at least the same size.

- $\mathcal{S}$ contains an unlooping sequence around $v$ that starts and finishes in some childbranches at $v$, and passes through the parent-branch $Q$ at $v$. Let $\left(P_{1}, \cdots, P_{s}\right)$ be the unlooping sequence, let $i<s$ such that $P_{i}$ and $P_{i+1}$ intersect $Q$, and let $x$ (resp., $y$ ) be the end of $P_{i}$ (resp., $P_{i+1}$ ) in $Q$. Finally, let $Q^{\prime}$ be the component of $T-\{y\}$ that does not contain $v$ and let $Y=T-Q$.

By Lemma 2.16, $\mathcal{S}$ augments at most $2+\sum_{1 \leq i \leq h}\left\lfloor\left(x_{i}\right) / 2\right\rfloor$ paths in $Y$ and then computes independently an optimal solution of $Q^{\prime}$ augmenting $z$ paths. Since combining an optimal solution of $Q^{\prime}$ with the augmentation of the path between $x$ and $y$ provides a solution for $Q$, SparseTreeAlgorithm augments at least $z+1$ paths in $Q$. Overall, SparseTreeAlgorithm augments at least $2+z+\sum_{1<i<h}\left\lfloor\left(x_{i}\right) / 2\right\rfloor$ paths in $T$. Hence, the solution computed by the algorithm has at least the same size as the one of $\mathcal{S}$.

Case 7. In that case, there is an unlooping sequence $\mathcal{S}^{\prime}=\left(P_{1}, \cdots, P_{s}\right)$ of paths containing $v$, characterized by a sequence $\left(T_{1}, \cdots, T_{s+1}\right)$ of components of $T-\{v\}$. Moreover, $T_{1}$ and $T_{s+1}$ are odd child-branches at $v$, there exists $j \leq s$ such that $T_{j}$ is the parent-branch at $v$, and $T_{i}$ is an even child-branch at $v$ for every $1<i \leq s, i \neq j$. Let $x$ be end of $P_{j-1}$ on $T_{j}$ and $y$ be the end of $P_{j}$ in $T_{j}$.

Also, there exists no unlooping sequence starting and finishing in some odd child-branches and avoiding the parent-branch. In the present case, SPARSETrEeAlgorithm first augments the paths in $\mathcal{S}^{\prime}$ and then the components of $T-\bigcup_{i \leq s} V\left(P_{i}\right)$ independently. Let $B_{1}, \cdots, B_{h}$ be the child-branches at $v$ and, for every $i \leq h$, let $x_{i}$ be the number of exposed nodes of $B_{i}$. Let $Q$ be the parent-branch at $v$. Said differently, SparseTreeAlgorithm computes an optimal solution for $Q \backslash P_{j}$ (by induction) and, by Lemma 2.16, augments $2+\sum_{i \leq h}\left\lfloor x_{i} / 2\right\rfloor$ paths intersecting $T_{v}$. Let us compare this number of augmentations with the one obtained by the optimal sequence $\mathcal{S}$. There are several cases to be considered.

- No path of $\mathcal{S}$ contains $v$ and some node of a child-branch at $v$. Note that, since $\mathcal{S}^{\prime}$ exists, $v$ was initially matched by $M$ with a node in one of its child-branches. Therefore, $\mathcal{S}$ cannot contain either a path that contains $v$ and some node of the parent-branch at $v$. Hence, $\mathcal{S}$ deals with $Q$ and $T_{v}-\{v\}$ independently. By Lemma 2.16, at most $\sum_{i \leq h}\left\lfloor x_{i} / 2\right\rfloor$ paths may be augmented. Moreover, by Lemma 2.12 (applied twice: once for $x$ and once for $y$ ), in $Q$, the number of paths that may be augmented is at most the maximum number of paths that may be augmented in $Q \backslash P_{j}$ plus 2 . Overall, $\mathcal{S}$ augments at most the same number of paths as SPARSETREeAlgorithm.

So, because no unlooping sequence avoiding the parent-branch exists, if $\mathcal{S}$ contains paths intersecting $v$, it must be via unlooping sequences intersecting the parent-branch.

- $\mathcal{S}$ contains an unlooping sequence around $v$ that starts or finishes in the parent-branch at $v$ (in particular, it starts or finishes in $x$ ). Then, by Lemma 2.16, $\mathcal{S}$ augments at most $1+\sum_{1<i<h}\left\lfloor\left(x_{i}\right) / 2\right\rfloor$ paths intersecting $T_{v}$ and then computes an optimal solution in the subtree $\bar{Q}^{\prime}$ of $Q$ (precisely the component of $Q \backslash\{x\}$ containing $y$ ). By Lemma 2.12 (applied to $y$ ), an optimal solution of $Q^{\prime}$ is at most 1 plus an optimal solution for $Q \backslash P_{j}$. Therefore, the solution computed by SpARSETREeAlgorithm has at least the same size.

- Finally, let us assume that $\mathcal{S}$ contains an unlooping sequence around $v$ that starts and finishes in some child-branches at $v$, and passes through the parent-branch $Q$ at $v$. By Lemma 2.16, $\mathcal{S}$ augments at most $2+\sum_{1 \leq i \leq h}\left\lfloor\left(x_{i}\right) / 2\right\rfloor$ paths and, independently, 
computes an optimal solution in $Q \backslash P_{j}$. So $\mathcal{S}$ does the same as SparseTreeAlgoRITHM (possibly augmenting a different unlooping sequence around $v$ ). Hence, SparseTreeAlgorithm obtains a solution of same size.

Case 8. In that case, there is an unlooping sequence $\mathcal{S}^{\prime}=\left(P_{1}, \cdots, P_{s}\right)$ of paths containing $v$, characterized by a sequence $\left(T_{1}, \cdots, T_{s+1}\right)$ of components of $T-\{v\}$. Moreover, $T_{1}$ is an odd child-branch at $v, T_{2}, \cdots, T_{s}$ are even child-branches at $v$, and $T_{s+1}$ is the parent-branch a $v$ (the case when $\mathcal{S}^{\prime}$ starts by the parent-branch is similar). Let $x$ be an end of $P_{s}$ in $T_{s+1}$.

There exists no unlooping sequence starting or finishing in some odd child-branch and that does not finishes or starts in the parent-branch. SparseTreeAlgorithm first augments the paths in $\mathcal{S}^{\prime}$ and then the components of $T-\bigcup_{i \leq s} V\left(P_{i}\right)$ independently. Let $B_{1}, \cdots, B_{h}$ be the child-branches at $v$ and, for every $i \leq h$, let $x_{i}$ be the number of exposed nodes of $B_{i}$. Let $Q$ be the parent-branch at $v$. Said differently, SparseTreeAlgorithm computes an optimal solution for $Q \backslash P_{s}$ (by induction) and, by Lemma 2.16, augments $1+\sum_{i<h}\left\lfloor x_{i} / 2\right\rfloor$ paths intersecting $T_{v}$. Let us compare this number of augmentations with the one obtained by the optimal sequence $\mathcal{S}$. There are several cases to be considered.

- No path of $\mathcal{S}$ contains $v$ and some node of a child-branch at $v$. Note that, since $\mathcal{S}^{\prime}$ exists, $v$ was initially matched by $M$ with a node in one of its child-branches. Therefore, $\mathcal{S}$ cannot contain a path that contains $v$ and some node of the parent-branch at $v$. Hence, $\mathcal{S}$ deals with $Q$ and $T_{v}-\{v\}$ independently. By Lemma 2.16 , at most $\sum_{i<h}\left\lfloor x_{i} / 2\right\rfloor$ paths may be augmented. Moreover, by Lemma 2.12 (applied once for $x$ ), in $Q$, the number of paths that may be augmented is at most the maximum number of paths that may be augmented in $Q \backslash P_{j}$ plus 1. Overall, $\mathcal{S}$ augments at most the same number of paths as SparseTreeAlgorithm.

- Now, because of the hypothesis, if $\mathcal{S}$ contains paths intersecting $v$, then we may assume that $\mathcal{S}$ contains an unlooping sequence around $v$ that starts or finishes in the parentbranch at $v$ (in particular, it starts or finishes in $x$ ). Then, by Lemma 2.16, $\mathcal{S}$ augments at most $1+\sum_{1<i<h}\left\lfloor\left(x_{i}\right) / 2\right\rfloor$ paths intersecting $T_{v}$ and then computes an optimal solution in the subtree $Q \backslash P_{s}$. Thus, SparseTreeAlgorithm obtains a solution of same size.

Case 9. In the last case, no unlooping sequence starting or finishing by an odd child-branch at $v$ exists. In that case, SparseTreeAlgorithm deals independently with $T_{v}-\{v\}$, in which case, by Lemma 2.16, it augments $\sum_{i \leq h}\left\lfloor x_{i} / 2\right\rfloor$ paths, and then computes an optimal (by induction) solution for $\left(T-T_{v}\right) \cup\{v\}$. As previously, it can be checked that no optimal sequence can augment strictly more paths.

\subsection{Caterpillars}

Recall that a caterpillar is a tree consisting of one main path (which we call spine throughout), such that every node either belongs to that path, or is adjacent to a node of that path. Prior to exhibiting a polynomial-time algorithm to solve $M P \leq k$ for caterpillars, we first need to show that a maximum matching can be attained by performing specific augmentations.

We start by pointing out that, in our context, we may consider caterpillars with maximum degree at most 3 . This follows from the following more general statement, which actually concerns all trees.

Lemma 2.18. Let $T$ be a tree, $M$ be a matching, and $v$ be a node of $T$ adjacent to $d \geq 2$ leaves $u_{1}, \cdots, u_{d}$. Then, for any sequence of $(\leq k)$-augmentations starting from $M$, at most one of the $u_{i}$ 's can be an end of an augmented path.

Proof. This follows from the fact that once a leaf gets covered by a matching, it cannot loose this property, recall Claim 2.1. Furthermore, once a leaf is covered, there is no more augmenting path containing $v$.

Let $C$ be a caterpillar, and $M$ be a matching of $C$. We denote by $\tilde{C}$ a caterpillar obtained from $C$ by removing some leaves, as follows. For every non-leaf node $v$ of the spine of $C$ : 
- if $v$ is adjacent to a covered leaf $u$, then we remove all leaves adjacent to $v$, but $u$;

- otherwise, we remove all leaves adjacent to $v$, but one.

From Lemma 2.18, we deduce the following.

Corollary 2.19. Let $C$ be a caterpillar, and $M$ be a matching. Let $M^{\prime}$ be the restriction of $M$ to $\tilde{C}$. Then $\mu_{\leq k}(C, M)=\mu_{\leq k}\left(\tilde{C}, M^{\prime}\right)$.

As a consequence of Corollary 2.19, we may narrow down our attention on caterpillars with maximum degree 3, i.e., we may assume that every node of the spine is adjacent to at most one leaf. Therefore, we may denote the nodes of any considered caterpillar $C$ in the following way. Let $\left(u_{1}, \cdots, u_{\ell}\right)$ be the spine of $C$ (where $u_{1}$ and $u_{\ell}$ are leaves of $T$ ). For any $i \in\{2, \cdots, \ell-1\}$ we denote by $u_{i}^{\prime}$ the leaf (not belonging to the spine) adjacent to $u_{i}$, if it exists. Note that $u_{2}^{\prime}$ and $u_{\ell-1}^{\prime}$ do not exist. Note that this labelling of the nodes is not consistent for the subcaterpillars of $C$. That is, when removing a node $u_{i}$ of $C$, the node $u_{i+1}^{\prime}$ might belong to the spine of one connected component of $C-\left\{u_{i}\right\}$. Hence, in what follows, for every caterpillar we consider, we implicitly assume that the labelling of its nodes respect the convention above.

We start by pointing out that, in a caterpillar $C$ with maximum degree 3 , starting from a matching $M$, a matching with size $\mu_{\leq k}(C, M)$ can be obtained by performing non-intersecting $(\leq k)$-augmentations.

Lemma 2.20. Let $C$ be a caterpillar with maximum degree $3, M$ be a matching, and $\mathcal{S}=$ $\left(P_{1}, \cdots, P_{r}\right)$ be a sequence of $(\leq k)$-augmentations starting from $M$. Assume $P_{i}$ and $P_{i+1}$ intersect, for some $i<r$. Then, there exist two disjoint paths $A, B$ such that $\left(P_{1}, \cdots, P_{i-1}, A, B, P_{i+2}, \cdots, P_{r}\right)$ is a sequence of $(\leq k)$-augmentations resulting in the same matching as $\mathcal{S}$.

Proof. This follows from arguments that are similar to that we used to prove Lemma 2.7 (with the exception that, here, it might be that none of $P_{i}$ and $P_{i+1}$ strictly includes the other). Consider, as $A$ and $B$, the exactly two paths of $\left(P_{i} \cup P_{i+1}\right)-E\left(P_{i} \cap P_{i+1}\right)$. Note that if $P_{i}$ and $P_{i+1}$ are vertexdisjoint, then $\{A, B\}=\left\{P_{i}, P_{i+1}\right\}$. It can be checked that $A$ and $B$ are disjoint, and, because $C$ is a caterpillar, that they have length at most $k$ (because $P_{i}$ and $P_{i+1}$ do). More precisely, each of these paths $A$ and $B$ is either:

- a subpath of $P_{i+1}$, or

- obtained from $P_{i}$ or $P_{i+1}$ by replacing a subpath of $P_{i}$ (hence of length at least 1) and a leaf edge (in $C$ ) of $P_{i+1}$.

Furthermore, executing $\left(P_{1}, \cdots, P_{i-1}, A, B, P_{i+2}, \cdots, P_{r}\right)$ from $M$ results in the same matching as $\mathcal{S}$.

Let $C$ be a caterpillar with maximum degree 3 and $M$ be a matching. If $u_{1}$ is covered by $M$ then no augmenting path will never contain $u_{1}$ nor $u_{2}$ (which is also obviously covered). Hence, removing $u_{1}$ and $u_{2}$ (recall that $u_{2}^{\prime}$ does not exist) from $C$ and removing $u_{1} u_{2}$ from $M$ results in a new instance $\left(C^{\prime}, M^{\prime}\right)$ such that solving the problem in $\left(C^{\prime}, M^{\prime}\right)$ is equivalent to solving the problem in $(C, M)$. Therefore, we may assume that $u_{1}$, which is a leaf, is exposed. When referring to the closest exposed node of $u_{1}$ in $C$, we mean the exposed node (different from $u_{1}$ ) that is at shortest distance from $u_{1}$. Note that there may be two candidates $u_{i}^{\prime}$ and $u_{i+1}$ (for some $i>1$ ) as such closest exposed nodes. In that case, we consider $u_{i}^{\prime}$ as the closest exposed node from $u_{1}$. By definition, the path from $u_{1}$ to its closest exposed node is augmenting. Furthermore, we may suppose that $u_{1}$ and its closest exposed node are at distance at most $k$. Otherwise, no augmenting path of length at most $k$ can never contain vertices in $\left\{u_{1}, \cdots, u_{i}, u_{i}^{\prime}\right\}$. In such case (if $u_{i}^{\prime}$ is at distance more than $k$ from $u_{1}$ ), $C$ and $M$ could be simplified by removing all vertices in $\left\{u_{1}, \cdots, u_{i}, u_{i}^{\prime}\right\}$, and applying the algorithm on the remaining caterpillar. Now, by the left-most augmentation in $C$, we refer to the augmentation joining $u_{1}$ and its closest exposed node.

From Lemma 2.20, we now prove that, for a caterpillar $C$ and a matching $M$, we can obtain, via $(\leq k)$-augmentations, a matching of size $\mu_{\leq k}(C, M)$ by repeatedly performing left-most augmentations. 
Lemma 2.21. Let $C$ be a caterpillar with maximum degree 3 and $M$ be a matching. Then, a matching of size $\mu_{\leq k}(C, M)$ can be obtained by repeatedly performing left-most $(\leq k)$-augmentations starting from $M$.

Proof. According to Lemma 2.20 and Claim 2.3, a matching of size $\mu_{\leq k}(C, M)$ can be obtained, starting from $M$, by a sequence $\mathcal{S}=\left(P_{1}, \cdots, P_{r}\right)$ of pairwise disjoint $(\leq k)$-augmentations. Furthermore, by Claim 2.3, we may assume that $P_{1}<\cdots<P_{r}$, i.e., that the right-most end of $P_{i}$ is located on the left of the left-most end of $P_{i+1}$, for every two consecutive $P_{i}, P_{i+1}$. To prove the lemma, it suffices to show that a matching of size $\mu_{\leq k}(C, M)$ can be obtained via a sequence $\left(P_{1}^{\prime}, P_{2}, \ldots, P_{r}\right)$ of $(\leq k)$-augmentations, where $P_{1}^{\prime}<P_{2}<\cdots<P_{r}$ and $P_{1}^{\prime}$ is the left-most augmentation. If $P_{1}$ is already the left-most augmentation, then we set $P_{1}^{\prime}=P_{1}$. Otherwise, let $P_{1}^{\prime}$ be the left-most $(\leq k)$-augmentation in the subcaterpillar containing all nodes located on the left of the right-most end of $P_{1}$. Then we just repeat, by induction, the same arguments with the subcaterpillar containing all nodes located on the right of the right-most end of $P_{1}^{\prime}$, and with the sequence $\left(P_{2}, \cdots, P_{r}\right)$ of $(\leq k)$-augmentations.

Corollary 2.19 and Lemma 2.21 directly yield a linear-time algorithm for solving $M P^{\leq k}$ in a caterpillar $C$. First, we may suppose that $C=\tilde{C}$ as otherwise the instance could be simplified. Then, a matching of size $\mu_{\leq k}(C, M)$ can be obtained by repeatedly applying the left-most $(\leq k)$ augmentation, for the notion of left and right we have used throughout this section.

Theorem 2.22. Let $C$ be a caterpillar, and $M$ be a matching. Then, $\mu_{\leq k}(C, M)$ can be computed in linear time (in $|V(C)|$ ).

\section{Augmenting matchings via $(=k)$-augmentations}

We now investigate the consequences, on $M P^{\leq k}$, of restricting ourselves to augmentations of paths with length exactly $k$. In Section 3.1, we start by showing that $M P^{=k}$ is, in general, NPhard for every $k \geq 3$. The case of trees is considered in Section 3.2, wherein we prove that, in this context, the problem is NP-hard when $k$ is part of the instance.

\subsection{Complexity of $M P^{=k}$ in general graphs (for fixed $k$ )}

First, we would like to point out that, in the reduction of [NSW15] for showing that $M P^{\leq k}$ is NP-hard for every $k \geq 5$, only $(=k)$-augmentations can actually be performed in the reduced graphs, which can be checked by considering the pairs of exposed vertices. Therefore, from this reduction we directly get the following.

Theorem 3.1. [NSW15] $M P^{=k}$ is NP-hard for every $k \geq 5$, even when restricted to instances where $G$ is a planar bipartite graph with maximum degree 3 .

Since $M P^{\leq 3}$ was shown to be polynomial-time solvable [NSW15], we do not get the NP-hardness of $M P^{=3}$ right away, in a similar way, and instead have to provide a proof.

Theorem 3.2. $M P^{=3}$ is NP-hard, even when restricted to instances where $G$ is a planar bipartite graph with maximum degree 3 and arbitrarily large girth.

Before going to the proof of Theorem 3.2, we first describe the gadgets to be used, as well as some of their behaviours. We start off with choice gadgets (see Figure 1 for an illustration). For any $\ell \geq 2$, the $\ell$-choice gadget is obtained from a path $\left(u_{\ell}, u_{\ell-1}, \cdots, u_{1}, v, w_{1}, \cdots, w_{\ell-1}, w_{\ell}\right)$ on $2 \ell+1$ vertices by 1 ) joining a new pendant vertex $u_{i}^{\prime}$ to $u_{i}$, for every $\left.i=1, \cdots, \ell, 2\right)$ joining a new pendant vertex $w_{i}^{\prime}$ to $w_{i}$, for every $i=1, \cdots, \ell$, then 3 ) joining a new pendant vertex $v^{\prime}$ to $v$, and 4) joining a new pendant vertex $v^{\prime \prime}$ to $v^{\prime}$. We call $v v^{\prime}$ the middle-edge of the choice gadget. Throughout this section, assuming it is clear which choice gadget we are dealing with, we refer to its vertices and edges using the terminology we have just introduced.

One property of interest of choice gadgets is the following:

Observation 3.3. Let $H$ be an $\ell$-choice gadget, and $M=\left\{v v^{\prime}\right\}$ be a matching of $H$. Then, for any matching $M^{\prime}$ of size $\mu_{=3}(H, M)$ obtained by performing (=3)-augmentations starting from $M$, we have either: 

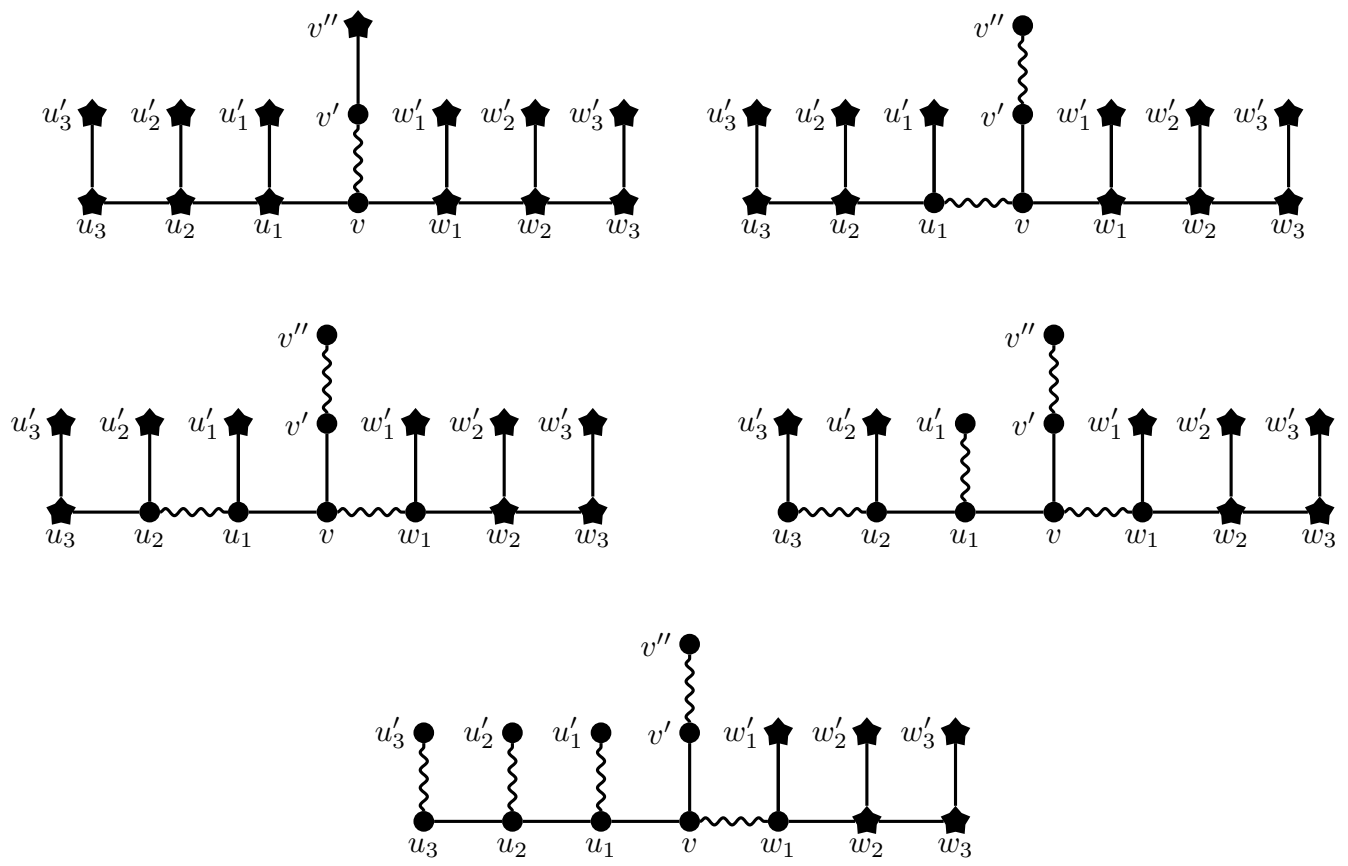

Figure 1: The 3-choice gadget $H$, and the way the initial matching $M=\left\{v v^{\prime}\right\}$ is being $(=3)$-augmented until a matching of size $\mu_{=3}(H, M)$ is attained (from top to bottom, and left to right). Star vertices are exposed vertices. Wiggly edges are edges of the matching.

1. $M^{\prime}=\left\{v^{\prime} v^{\prime \prime}\right\} \cup\left\{v w_{1}\right\} \cup \bigcup_{i=1}^{\ell}\left\{u_{i} u_{i}^{\prime}\right\}$ or

2. $M^{\prime}=\left\{v^{\prime} v^{\prime \prime}\right\} \cup\left\{v u_{1}\right\} \cup \bigcup_{i=1}^{\ell}\left\{w_{i} w_{i}^{\prime}\right\}$.

In particular, we have $\mu_{=3}(H, M)=\ell+2$.

Proof. Consider a maximum sequence $\mathcal{S}=\left(P_{1}, \cdots, P_{q}\right)$ of $(=3)$-augmentations that can be performed starting from $M$, and denote by $M^{\prime}$ the resulting matching. Throughout this proof, for every $1 \leq i \leq q$, we denote by $\mathcal{S}(M, i)$ the matching obtained from $M$ by augmenting sequentially the paths $P_{1}, \cdots, P_{i}$. Note that $\mathcal{S}(M, q)=M^{\prime}$.

Recall that a $(=3)$-augmentation can only be performed on a path of length 3 in which only the middle-edge belongs to the matching. For this reason, since $M=\left\{v v^{\prime}\right\}$, we have $P_{1}=\left(v^{\prime \prime}, v^{\prime}, v, u_{1}\right)$ or $P_{1}=\left(v^{\prime \prime} v^{\prime} v w_{1}\right)$. Assume $P_{1}=\left(v^{\prime \prime}, v^{\prime}, v, u_{1}\right)$ without loss of generality (the other case is symmetric). Because $v^{\prime \prime} v^{\prime} \in \mathcal{S}(M, 1)$ and $v^{\prime \prime}$ has degree 1 , no augmenting path can contain any of $v^{\prime \prime}$ and $v^{\prime}$. So $P_{2}$ necessarily contains $w_{1} v$ and $v u_{1}$. More precisely, $P_{2}$ may be either $\left(w_{1}, v, u_{1}, u_{2}\right)$ or $\left(w_{1}, v, u_{1}, u_{1}^{\prime}\right)$. At this point, it can be noted that, since $v w_{1}$ and $v u_{1}$ belong to one of $P_{1}$ and $P_{2}$, they cannot belong to any $P_{i}$ with $i \geq 3$. Indeed, whatever be the choice of $P_{2}$, after its augmentation, all the neighbours of $v$ get covered, and, thus, no edge incident to $v$ may belong to an augmenting path of length 3 from this point. This implies that none of $w_{2}$ and $w_{1}^{\prime}$ belongs to one of the $P_{i}$ 's, thus that the matching cannot be spread further towards the $w_{i}$ 's. We also note that if $P_{2}=\left(w_{1}, v, u_{1}, u_{1}^{\prime}\right)$, then, in $H$ and $\mathcal{S}(M, 2)$, there is no further (=3)-augmentation, which means that the matching cannot propagate further towards the $u_{i}$ 's. So we necessarily have $P_{2}=\left(w_{1}, v, u_{1}, u_{2}\right)$.

We thus have $\mathcal{S}(M, 2)=\left\{v^{\prime} v^{\prime \prime}, v w_{1}, u_{1} u_{2}\right\}$, and the only augmenting (=3)-paths are $\left(u_{1}^{\prime}, u_{1}, u_{2}, u_{2}^{\prime}\right)$ and $\left(u_{1}^{\prime}, u_{1}, u_{2}, u_{3}\right)$ (if $\ell \geq 3$ ). If $\ell=2$, then necessarily $P_{3}=\left(u_{1}^{\prime}, u_{1}, u_{2}, u_{2}^{\prime}\right)$, and we are done. If $\ell>3$, we note that $P_{3}$ cannot be $\left(u_{1}^{\prime}, u_{1}, u_{2}, u_{2}^{\prime}\right)$ as otherwise $\mathcal{S}(M, 3)$ would have no augmenting (=3)-paths in $H$. So, in that case, $P_{3}=\left(u_{1}^{\prime}, u_{1}, u_{2}, u_{3}\right)$. These arguments generalize as follows by induction on $i$ (see Figure 1 for an illustration). For every $i=2, \cdots, \ell+1$, the only (=3)-augmenting paths in $H$, assuming the current matching is $\mathcal{S}(M, i-1)$, are $\left(u_{i-1}^{\prime}, u_{i-1}, u_{i}, u_{i}^{\prime}\right)$ and $\left(u_{i-1}^{\prime}, u_{i-1}, u_{i}, u_{i+1}\right)$. In case $i=\ell+1$, actually only the first of these two paths exists, so $P_{i}=\left(u_{i-1}^{\prime}, u_{i-1}, u_{i}, u_{i}^{\prime}\right)$. Otherwise, i.e., $i=2, \cdots, \ell$, we note that having $P_{i}=\left(u_{i-1}^{\prime}, u_{i-1}, u_{i}, u_{i}^{\prime}\right)$ 


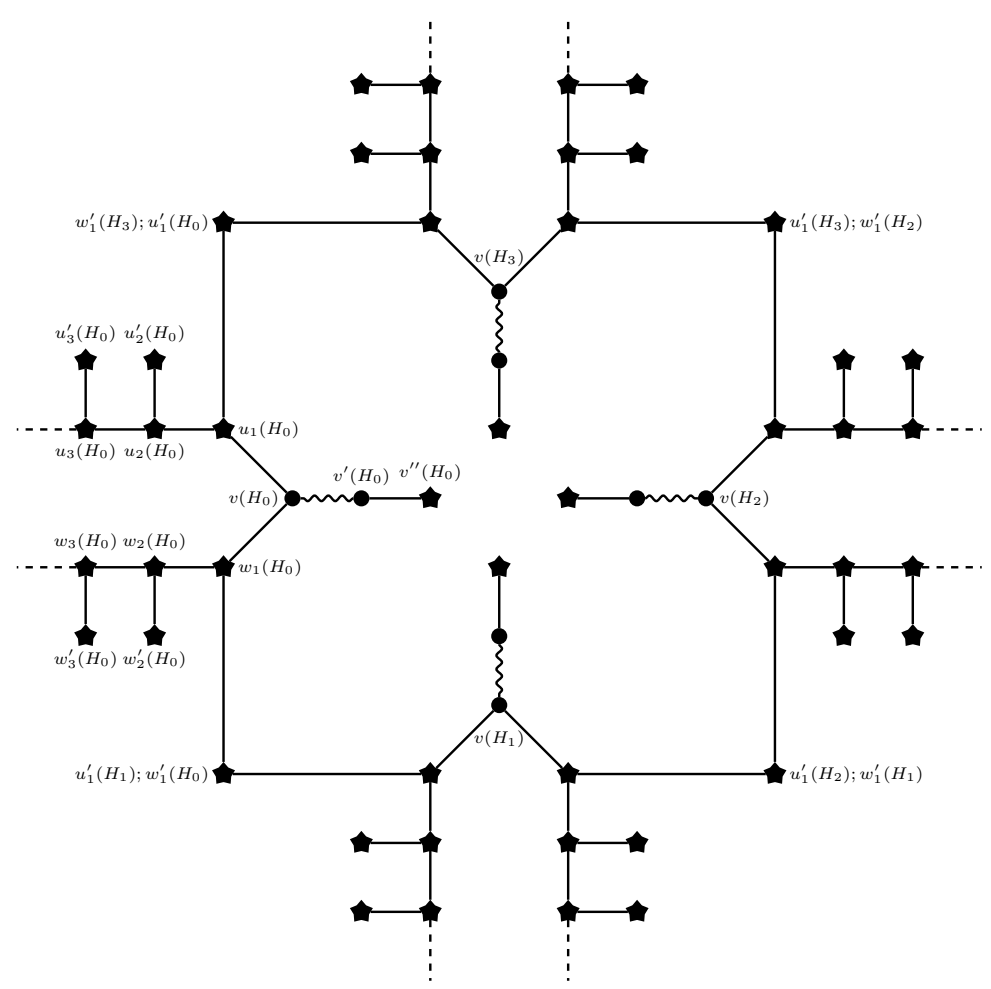

Figure 2: The 4-variable gadget. Star vertices are exposed vertices. Wiggly edges are edges of the matching.

would make $\mathcal{S}(M, i)$ have no augmenting $(=3)$-paths in $H$. So we have $P_{i}=\left(u_{i-1}^{\prime}, u_{i-1}, u_{i}, u_{i+1}\right)$ for every $i=2, \cdots, \ell$, so that the matching can be spread further.

Under the assumption that $P_{1}=\left(v^{\prime \prime}, v^{\prime}, v, u_{1}\right)$, we eventually get, assuming that $\mathcal{S}$ is maximum, that $M^{\prime}=\left\{v^{\prime} v^{\prime \prime}\right\} \cup\left\{v w_{1}\right\} \cup \bigcup_{i=1}^{\ell}\left\{u_{i} u_{i}^{\prime}\right\}$. By symmetry, we note that having $P_{1}=\left(v^{\prime \prime}, v^{\prime}, v, w_{1}\right)$ results in $M^{\prime}=\left\{v^{\prime} v^{\prime \prime}\right\} \cup\left\{v u_{1}\right\} \cup \bigcup_{i=1}^{\ell}\left\{w_{i} w_{i}^{\prime}\right\}$. In both cases, we have $\mu_{=3}(H, M)=\ell+2$, as claimed.

In order to introduce the next type of gadgets, we need some additional terminology to deal with choice gadgets. Let $H$ be a choice gadget. The vertices $u_{i}^{\prime}$ of $H$ are called the spike vertices of $H$, while the edges $u_{i} u_{i}^{\prime}$ incident to the spike vertices are called the spike edges. Each spike vertex or edge is numbered accordingly to the index $i$ of the vertex $u_{i}^{\prime}$ (or $w_{i}^{\prime}$ ) it intersects. The vertices $u_{1}, u_{1}^{\prime}, u_{2}, u_{2}^{\prime}, \cdots$ form the positive branch of $H$, while the vertices $w_{1}, w_{1}^{\prime}, w_{2}, w_{2}^{\prime}, \cdots$ form the negative branch. Rephrased differently, Observation 3.3 says that, in a choice gadget, the "optimal" way to propagate the original matching is towards the spike edges of the positive branch only, or towards the spike edges of the negative branch only. In the first case, we say that the original matching has been propagated positively, while we say it has been propagated negatively otherwise.

We now introduce variable gadgets, that are combinations of choice gadgets connected in a cyclic fashion (see Figure 2 for an illustration). For any $m \geq 1$, the $m$-variable gadget is constructed as follows. The 1 -variable gadget is the $\ell$-choice gadget (the length $\ell$ of the choice gadget will be fixed later). Then, for any $m \geq 2$, the $m$-variable gadget is constructed by taking $m \ell$-choice gadgets $H_{0}, \cdots, H_{\ell-1}$ and, for every $i=0, \cdots, \ell-1$, by identifying the first negative spike vertex of $H_{i}$ and the first positive spike vertex of $H_{i+1}$, where the indexes are understood modulo $m$. Precisely, for any $j<m$ and any $i \leq \ell$, let, here and further, $u_{i}\left(H_{j}\right)$ denote the vertex $u_{i}$ of the $j^{t h}$ copy $H_{j}$ of the choice gadget $\left(v\left(H_{j}\right), v^{\prime}\left(H_{j}\right), v^{\prime \prime}\left(H_{j}\right), u_{i}^{\prime}\left(H_{j}\right), w_{i}\left(H_{j}\right), w_{i}^{\prime}\left(H_{j}\right)\right.$ are defined analogously). Hence, the variable gadget is obtained by identifying $w_{1}^{\prime}\left(H_{j}\right)$ with $u_{1}^{\prime}\left(H_{j+1}\right)$ (modulo $m$ ) for every $j=0, \cdots, m-1$.

The original matching of any variable gadget is the union of the original matchings of the choice gadgets constituting it (i.e., their middle-edges). By the positive branches (resp. negative 
branches) of $H$, we mean the $m$ positive (resp. negative) branches of its underlying choice gadgets. Analogously, by referring to the spike vertices and spike edges of a variable gadget, we mean the spike vertices and edges of its underlying choice gadgets.

Note that we have not explicited the lengths of the $2 m$ branches composing an $m$-variable gadget, i.e., $\ell$ still must be defined. However, we must now ensure that the behaviour described in Observation 3.3 remains valid after having combined the choice gadgets to form a variable gadget. Assuming these branches are "long enough", we prove it is the case.

Observation 3.4. Let $H$ be an $m$-variable gadget, and $M$ be the original matching of $H$ as described above. If $\ell$ (the length of the choice gadgets composing $H$ ) is at least 2 , then, for any matching $M^{\prime}$ of size $\mu_{=3}(H, M)$ obtained by performing $(=3)$-augmentations starting from $M$, we have either:

1. all positive spike edges in $M^{\prime}$ and no negative spike edges in $M^{\prime}$, or

2. all negative spike edges in $M^{\prime}$ and no positive spike edges in $M^{\prime}$.

Proof. Let $H_{0}, \cdots, H_{m-1}$ be the $m \ell$-choice gadgets composing $H$. We first prove that, because $\ell \geq$ 2 , any maximum sequence of $(=3)$-augmentations has no interest to perform $(=3)$-augmentations that intersect different choice gadgets. That is, a matching of size $\mu_{=3}(H, M)$ cannot be obtained by augmenting a $(=3)$-path having edges in two consecutive $H_{i}$ 's.

For purpose of contradiction, let us assume that there is an augmenting $(=3)$-path $Q$ that intersects two choice gadgets, w.l.o.g., say $H_{0}$ and $H_{1}$ and that $Q$ has two edges in $H_{0}$. Let us assume that $Q$ is the first (to be augmented) such path intersecting distinct choice gadgets. When $Q$ is about to be augmented, $w_{1}\left(H_{0}\right) w_{1}^{\prime}\left(H_{0}\right)$ must be in the current matching, a neighbour of $w_{1}\left(H_{0}\right)$ must be exposed, and $u_{1}\left(H_{1}\right)$ must be exposed. The only way to have reached such a situation is when, in $H_{0}$, the paths $\left(v^{\prime \prime}\left(H_{0}\right), v^{\prime}\left(H_{0}\right), v\left(H_{0}\right), w_{1}\left(H_{0}\right)\right)$ and $\left(u_{1}\left(H_{0}\right), v\left(H_{0}\right), w_{1}\left(H_{0}\right), w_{1}^{\prime}\left(H_{0}\right)\right)$ have been augmented, and only them. Moreover, only the path $\left(v^{\prime \prime}\left(H_{1}\right), v^{\prime}\left(H_{1}\right), v\left(H_{1}\right), w_{1}\left(H_{1}\right)\right)$ can have been augmented in $H_{1}$.

Therefore, $Q$ must be $\left(w_{2}\left(H_{0}\right), w_{1}\left(H_{0}\right), w_{1}^{\prime}\left(H_{0}\right), u_{1}\left(H_{1}\right)\right)$. After having augmented $Q$, we note that, because all of $v^{\prime \prime}\left(H_{0}\right), v^{\prime}\left(H_{0}\right), v\left(H_{0}\right), u_{1}\left(H_{0}\right), w_{1}\left(H_{0}\right) w_{2}\left(H_{0}\right), w_{1}^{\prime}\left(H_{0}\right)$ are covered, there is, in $H$, no further $(=3)$-augmentation including an edge of $H_{0}$. In $H_{1}$, we note that, since $u_{1}^{\prime}\left(H_{1}\right)$ and $u_{1}\left(H_{1}\right)$ are covered, the only $(=3)$-augmentation that can potentially be performed (if not already) in $H_{1}$ is $\left(v^{\prime \prime}\left(H_{1}\right), v^{\prime}\left(H_{1}\right), v\left(H_{1}\right), w_{1}\left(H_{1}\right)\right)$. According to these arguments, augmenting a (=3)-path intersecting $H_{0}$ and $H_{1}$ leads to a final matching with at most three edges in $H_{0}$ and three edges in $H_{1}$, while by Observation 3.3 we could have achieved a matching with $\ell+2 \geq 4$ edges in each of $H_{0}$ and $H_{1}$ by propagating the matching positively or negatively.

Following these observations, since $\ell \geq 2$, a matching of size $\mu_{=3}(H, M)$ can only be obtained, from $M$, by propagating the original matching of each $H_{i}$ positively or negatively. The claim now follows from the fact that if, say, $H_{i}$ has propagated its matching negatively, then $H_{i+1}$ cannot propagate its matching positively, since the first negative spike edge of $H_{i}$ and the first positive spike edge of $H_{i+1}$ are adjacent.

According to Observation 3.4, we can thus derive the notion of positive and negative propagations to variable gadgets with long branches: by propagating its matching positively (resp. negatively), we mean propagating the matching positively (resp. negatively) in all of its underlying choice gadgets.

We now have all ingredients in hand for proving Theorem 3.2.

Proof of Theorem 3.2. The proof is by reduction from 3-SAT. Namely, from a 3CNF formula $\Phi$, we construct, in polynomial time, a graph $G$ with an initial matching $M$ such that, from a satisfiable truth assignment of the variables of $\Phi$, we can deduce a matching of size $\mu_{=3}(G, M)$ to which $M$ can be (=3)-augmented, and vice versa.

The construction of $G$ (and $M$ ) is mainly achieved by connecting several variable gadgets together. For every variable $x_{i}$ of $\Phi$, we add an $m_{i}$-variable gadget $G_{i}$ to $G$, where

$$
m_{i}=\max \left\{\# \text { of distinct clauses that contain } x_{i}, \# \text { of distinct clauses that contain } \overline{x_{i}}\right\} \text {, }
$$




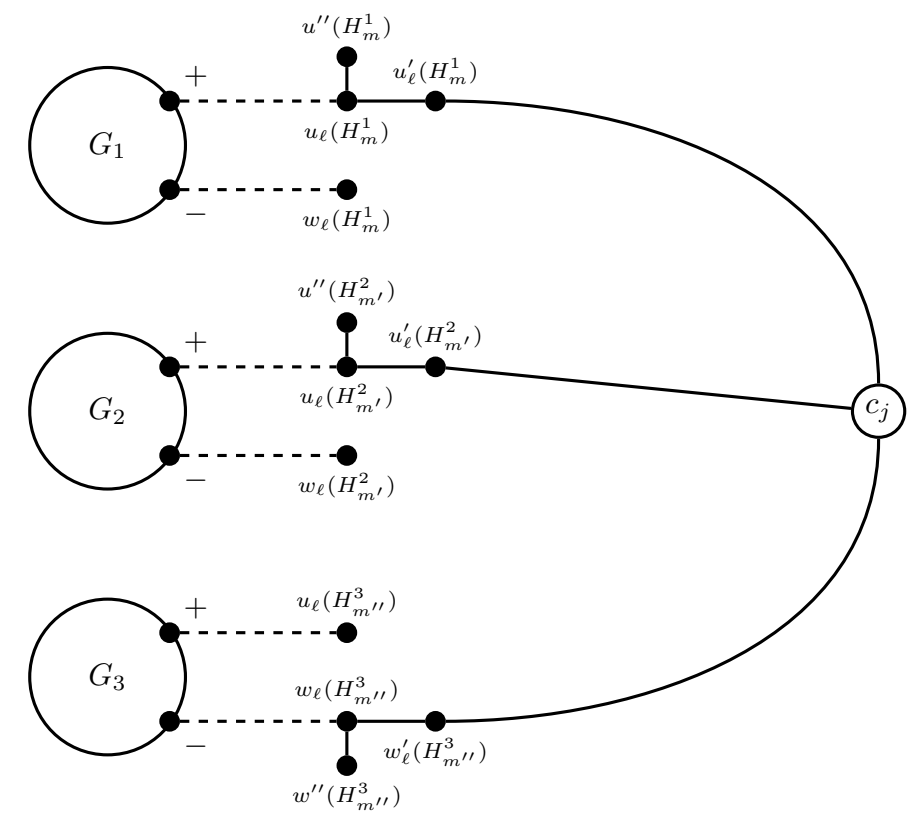

Figure 3: Illustration of the reduction in the proof of Theorem 3.2, for a formula $\Phi$ having a clause $C=\left(x_{1} \vee x_{2} \vee \overline{x_{3}}\right)$, and $C$ is the $m^{t h}$ (resp. $m^{\prime t h}, m^{\prime \prime t h}$ ) clause containing $x_{1}$ (resp. $\left.x_{2}, \overline{x_{3}}\right)$.

and the choice gadgets underling $G_{i}$ have length $\ell$ at least 2 (so that Observation 3.4 can apply). Let $H_{0}^{i}, \cdots, H_{m_{i}-1}^{i}$ be the choice gadgets composing $G_{i}$. For every last (i.e., the farthest from the middle-edge) positive (resp. negative) spike edge $u_{\ell}\left(H_{j}^{i}\right) u_{\ell}^{\prime}\left(H_{j}^{i}\right)\left(\operatorname{resp} . w_{\ell}\left(H_{j}^{i}\right) w_{\ell}^{\prime}\left(H_{j}^{i}\right)\right)$ of every choice gadget $H_{j}^{i}$ underlying $G_{i}$, we add a pendant vertex $u^{\prime \prime}\left(H_{j}^{i}\right)$ (resp. $\left.w^{\prime \prime}\left(H_{j}^{i}\right)\right)$ that we join to $u_{\ell}\left(H_{j}^{i}\right)\left(\operatorname{resp.} w_{\ell}\left(H_{j}^{i}\right)\right)$.

For every clause $C_{j}$ of $\Phi$, we add a clause vertex $c_{j}$ to $G$. Finally, we connect the variable gadgets and clause vertices in the following way (see Figure 3): for every variable $x_{i}$ (resp. negated variable $\overline{x_{i}}$ ) and $m^{\text {th }}$ distinct clause $C_{j}$ containing $x_{i}$ (resp. $\overline{x_{i}}$ ), we join $c_{j}$ and the spike vertex $u_{\ell}^{\prime}\left(H_{m}^{i}\right)$ (resp. $\left.w_{\ell}^{\prime}\left(H_{m}^{i}\right)\right)$ from the $m^{t h}$ choice gadget $H_{m}^{i}$ of $G_{i}$. Note that the order in which the clauses containing $x_{i}$ are taken is not relevant.

We eventually define the original matching $M$ of $G$ as the union of the original matchings of the choice gadgets composing the variable gadgets. Clearly, the construction of $G$ is achieved in polynomial time.

A matching $M^{\prime}$ of size $\mu_{=3}(G, M)$ of $G$ that can be obtained starting from $M$ via $(=3)$ augmentations, is obtained as follows. Recall that the choice gadgets constituting the variable gadgets are assumed long, so that Observation 3.4 applies. First, for every variable gadget $G_{i}$, we have to propagate the original matching of $G_{i}$ either positively or negatively. Thus, at this point, the maximum number of such $(=3)$-augmentations that can be performed does not depend on the connexion with the clause vertices, but only on the lengths of the choice gadgets we used. So, from now on, we denote by $\alpha_{M}$ this number of $(=3)$-augmentations. That is, by Observations 3.3 and 3.4, we have

$$
\alpha_{M}=\sum_{i=1}^{n} m_{i} \cdot(\ell+2)
$$

where $n$ is the number of variables in $\Phi$. Now, for every clause $C_{j}$ of $\Phi$, we can potentially augment one of the at most three (= 3)-paths of the form $\left(c_{j}, u_{\ell}^{\prime}\left(H_{m}^{i}\right), u_{\ell}\left(H_{m}^{i}\right), u^{\prime \prime}\left(H_{m}^{i}\right)\right)$ or $\left(c_{j}, w_{\ell}^{\prime}\left(H_{m}^{i}\right), w_{\ell}\left(H_{m}^{i}\right), w^{\prime \prime}\left(H_{m}^{i}\right)\right)$ joined to $c_{j}$ in $G$ (where $m$ is the index of the choice gadget of $G_{i}$ that is associated to clause $C_{j}$ ), but this is only possible if one of the three variable gadgets incident to $c_{j}$ propagated its matching positively (first case), or negatively (second case). The matching then cannot be propagated further through $c_{j}$ : if, say, $u^{\prime \prime}\left(H_{m}^{i}\right) c_{j}$ belongs to the matching at some point, then it means that the $(=3)$-path $\left(c_{j}, u_{\ell}^{\prime}\left(H_{m}^{i}\right), u_{\ell}\left(H_{m}^{i}\right), u^{\prime \prime}\left(H_{m}^{i}\right)\right)$ was augmented, hence that $u_{\ell}^{\prime}\left(H_{m}^{i}\right)$ is covered while $u^{\prime \prime}\left(H_{m}^{i}\right)^{\prime}$ has degree 2 (so no $(=3)$-augmenting path including 
$u^{\prime \prime}\left(H_{m}^{i}\right) c_{j}$ exists). So we have

$$
\alpha_{M} \leq \mu_{=3}(G, M) \leq \alpha_{M}+\gamma,
$$

where $\gamma$ denotes the number of clauses appearing in $\Phi$. In particular, the upper bound is attained whenever, for every variable gadget $G_{i}$ of $G$, we can propagate its original matching appropriately, so that, for every clause vertex of $G$, an augmentation of an incident (=3)-path can be performed.

We claim that $\mu_{=3}(G, M)=\alpha_{M}+\gamma$ if and only if $\Phi$ admits a satisfying truth assignment. To see this holds, consider that, in $G$, propagating the original matching of any $G_{i}$ positively (resp. negatively) simulates the fact that variable $x_{i}$ of $\Phi$ is set to true (resp. false) by some truth assignment. So that one of the final $\gamma$ augmentations including the clause vertices of $G$ can be performed, it needs one of its at most three incident variable gadgets to have propagated its matching the good way. By this, we mean positively $(=t r u e)$ if the clause contains the positive version of that variable, of negatively $(=$ false $)$ otherwise. Hence, the equivalence holds.

We conclude the proof by pointing out some possible modifications of the reduction above, which maintain the equivalence with 3-SAT. Let us first point out that $G$ has maximum degree 3 . Furthermore, since 3-SAT remains NP-hard for planar formulas $\Phi$, we may assume that $\Phi$ is planar, in which case the reduced graph $G$ is clearly planar as well, since the variable gadgets are planar (as illustrated in Figure 2).

We can also modify the reduction above so that $G$ fulfils additional properties:

- It can be checked that all cycles of the reduced graph $G$ go through variable gadgets, i.e., at some point, they enter a variable gadget via a spike edge, and exit it using another spike edge (possibly from the other branch). Since two variable gadgets $G_{i}$ and $G_{i+1}$ are connected by spike edges or by clause vertices (hence by paths of length 2), and the positive and negative first (or last) spike edges of any $G_{i}$ are at even distance in $G_{i}$, the only way for a cycle of $G$ to be of odd length is when, in the construction, we use a choice gadget with odd length (so that, in its branches, its first and last spike edges are at odd distance). So, by using choice gadgets of even length only, we can make sure that $G$ is bipartite.

- Now, by increasing 1) the number of choice gadgets used to construct the $G_{i}$ 's, and 2) the length of these choice gadgets, we also increase the lengths of the cycles in $G$. So we can also make sure that $G$ has arbitrarily large girth.

\subsection{Complexity of $M P^{=k}$ in trees (for non-fixed $k$ )}

In this section, we show that $M P^{=k}$ is hard for trees when $k$ is part of the input. That is, we show that

(=)-Matching Problem $\left(M P^{=}\right)$

Input: A graph $G$, a matching $M$ of $G$, and an odd integer $k \geq 1$.

Question: What is the value of $\mu_{=k}(G, M)$ ?

is NP-hard, even for instances where $G$ is a tree.

Theorem 3.5. $M P^{=}$is NP-hard, even when restricted to instances where $G$ is a tree.

Proof. The proof is by reduction from 3-SAT. From a $3 \mathrm{CNF}$ formula $\Phi$, we construct a tree $T$ with a matching $M$, such that $\Phi$ is satisfiable if and only if $\mu_{=k}(T, M)$ is equal to a specific value, where $k$ depends on the number of variables and clauses of $\Phi$. For the sake of understanding, we here describe the proof for $k=100 \cdot(n+m)+1$, where $n$ is the number of distinct variables appearing in $\Phi$ and $m$ is the number of distinct clauses of $\Phi$, but the reduction could also be performed for much smaller values of $k$ (but still dependent on $n+m$ ). Note in particular that $\left\lfloor\frac{1}{10} k\right\rfloor$ is even.

The tree $T$, and its original matching $M$, are obtained by combining several gadgets together. These gadgets will each be equipped with an initial matching, so that $M$ will be the union of these matchings.

The first type of gadgets we use are departure gadgets of odd length $\ell \geq !$. The departure gadget is depicted in Figure 4 (left), where the wiggly edges are the edges of its original matching. The gadget consists of an augmenting path $\left(u_{\text {forth }}, x, w_{1}, \cdots, w_{\ell+1}\right)$ plus one node $u_{b a c k}$ adjacent to $w_{1}$. 

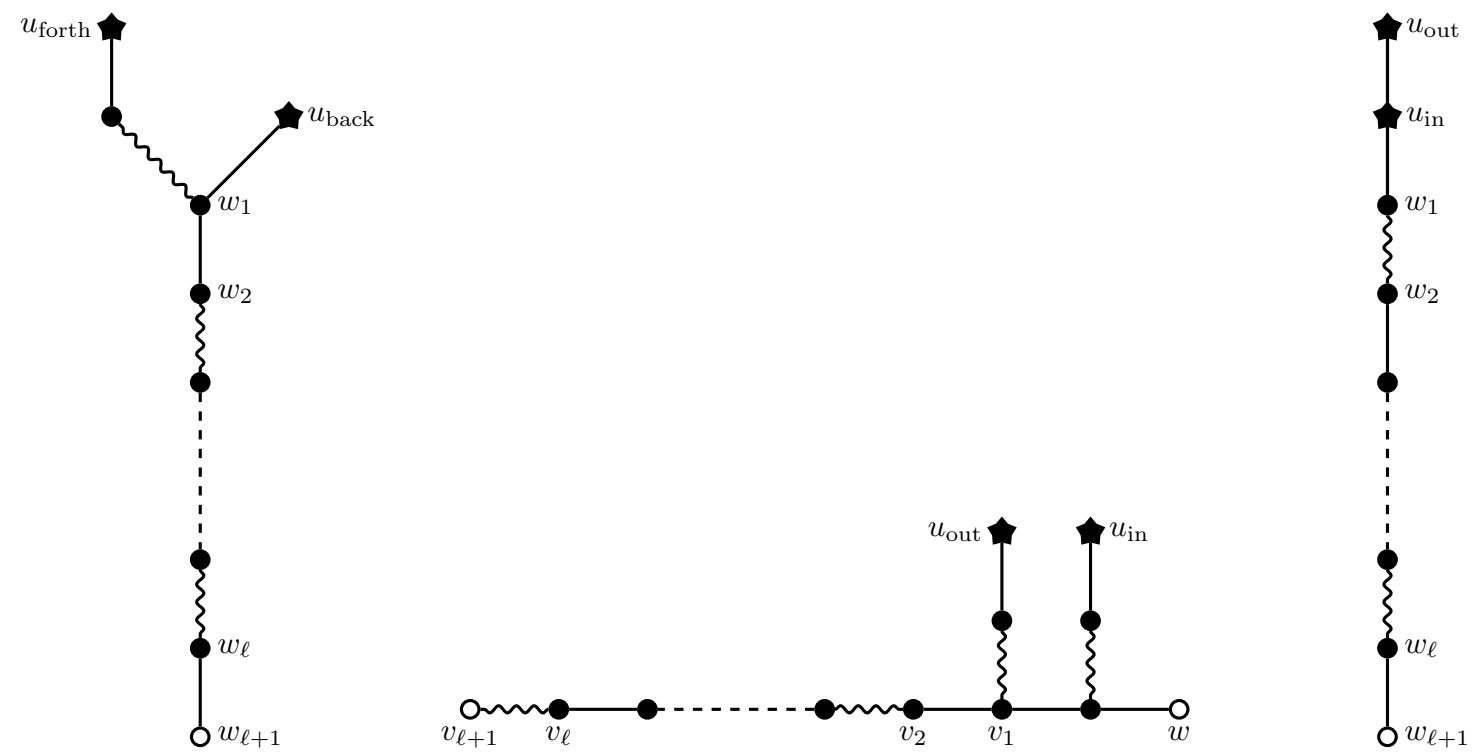

Figure 4: The departure gadget with length $\ell$ and root $w_{\ell+1}$ (left), the gate gadget with length $\ell$, first-root $w$, and second-root $v_{\ell+1}$ (middle), and the arrival gadget with length $\ell$ and root $w_{\ell+1}$ (right). Star nodes will be exposed in the reduced graph. White nodes (i.e., root nodes) will be used to attach the gadgets at some nodes. Wiggly edges are edges of the matching.

Its node $w_{\ell+1}$ is called the root of the gadget, as it will be used to attach the gadget to other gadgets. We call the two exposed nodes $u_{\text {forth }}$ and $u_{\text {back }}$ the forth-node and the back-node of the gadget, respectively, where the forth-node is the farther from $w_{\ell+1}$. The path from $w_{1}$ to $w_{\ell+1}$ might be of arbitrary even length $\ell$.

The second type of gadgets we need are gate gadgets of even length $\ell \geq 2$. This gadget is depicted in Figure 4 (middle). Its nodes $w$ and $v_{\ell+1}$ are the first-root and second-root, respectively, of the gadget. We call the exposed nodes $u_{\text {in }}$ and $u_{\text {out }}$ the in-node and the out-node, respectively. The path with even length $\ell$ from $v_{1}$ to $v_{\ell+1}$ is meant to be alternating. The initial matching of a gate gadget is the one depicted in Figure 4, namely the path from $v_{1}$ to $v_{\ell+1}$ has to be alternating with the second-root being covered, and the neighbours of the in- and out-nodes must be covered (while the in- and out-nodes must be exposed).

The last type of gadgets we need are arrival gadgets of even length $\ell \geq 2$, as depicted in Figure 4 (right). It is a path of even length $\ell+2$. The root of the gadget is $w_{\ell+1}$. The two exposed nodes $u_{\text {in }}$ and $u_{\text {out }}$ are called the in-node and out-node, respectively, of the gadget. The path from $w_{1}$ to $w_{\ell+1}$ is alternating so that $w_{1}$ is covered and $w_{\ell+1}$ is exposed.

We are now ready to describe how to combine these gadgets to form $T$ (and $M$ ); see Figure 5 for an illustration of the structure of $T$. We start from an edge $u v$, which we call the switch edge of $T$, and which belongs to $M$. We then add two special nodes $h_{v}$ and $h_{c}$ to $T$. We connect $h_{v}$ and $u$ (an end of the switch edge) via an alternating path with even length $\ell_{v}=\left\lfloor\frac{9}{10} k\right\rfloor$, in such a way that $h_{v}$ is covered by an edge of that path. Now, for every variable $x_{i}$ of $\Phi$, we identify $h_{v}$ and the root of a departure gadget $G\left(x_{i}\right)$ with odd length $\ell_{x_{i}}=2 i+1$. Note that, for every variable $x_{i}$, we have $\ell_{x_{i}}<\left\lfloor\frac{1}{10} k\right\rfloor$ by our choice of $k$. We do a similar construction for the clauses of $\Phi$. Namely, we connect $h_{c}$ and $u$ via an alternating path with even length $\ell_{c}=\left\lfloor\frac{2}{10} k\right\rfloor$, so that $h_{c}$ is covered by an edge of that path (and $u$ is not, since it is already covered by $u v$ ). Then, for every clause $C_{j}$ of $\Phi$, we identify $h_{c}$ and the root of a departure gadget $G\left(C_{j}\right)$ with odd length $\ell_{C_{j}}=2 j+1$. Here as well, note that $\ell_{C_{j}}<\left\lfloor\frac{1}{10} k\right\rfloor$ for every clause $C_{j}$.

We now consider every variable $x_{i}$ of $\Phi$ in turn. For every such $x_{i}$, we add, to $T$, a gate gadget $S\left(x_{i}\right)$ with even length $\ell_{x_{i}}^{\prime}=\left\lfloor\frac{6}{10} k\right\rfloor$. Similarly, we add to $T$ a gate gadget $S\left(\overline{x_{i}}\right)$ with even length $\ell_{\overline{x_{i}}}^{\prime}=\left\lfloor\frac{6}{10} k\right\rfloor$. We then identify the first roots of $S\left(x_{i}\right)$ and $S\left(\overline{x_{i}}\right)$. The resulting subgraph we have obtained for the couple $\left\{x_{i}, \overline{x_{i}}\right\}$ is denoted by $G\left(x_{i}, \overline{x_{i}}\right)$, and the node which served for connecting $S\left(x_{i}\right)$ and $S\left(\overline{x_{i}}\right)$ is called the root of $G\left(x_{i}, \overline{x_{i}}\right)$. We now connect the root of $G\left(x_{i}, \overline{x_{i}}\right)$ and $v$, the 


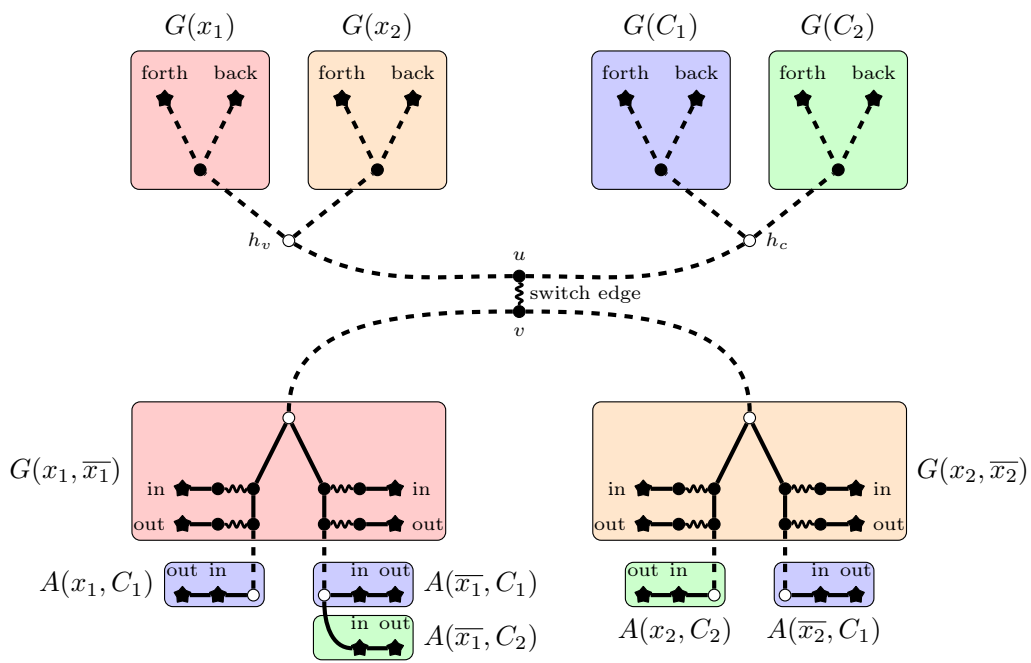

Figure 5: Illustration of the structure of $T$, as constructed in the proof of Theorem 3.5, for a formula $\Phi$ having two variables $x_{1}$ and $x_{2}$, and two clauses $C_{1}$ and $C_{2}$ such that $x_{1}, \overline{x_{1}}, \overline{x_{2}} \in C_{1}$ and $\overline{x_{1}}, x_{2} \in C_{2}$. Star nodes are exposed nodes. Wiggly edges are edges of the matching. The exposed nodes from an area with a given colour are only joined, via an augmenting $(=k)$-path, to another exposed node of the distinct area with the same colour.

end of the switch edge we have not used yet, via an alternating path with even length

$$
\ell_{x_{i}, \overline{x_{i}}}=k-\left(2+\ell_{x_{i}}+\ell_{v}+1+3\right)=k-\left(6+\ell_{x_{i}}+\left\lfloor\frac{9}{10} k\right\rfloor\right) .
$$

The initial matching on this path is made in such a way that the root of $G\left(x_{i}, \overline{x_{i}}\right)$ is covered by an edge of that path (and $v$ is not, since it is already covered by $u v$ ). Note that $\ell_{x_{i}, \overline{x_{i}}}<\left\lfloor\frac{1}{10} k\right\rfloor$ by our choice of $k$.

Now, for every clause $C_{j}$ of $\Phi$ containing $x_{i}$, we identify the second-root of $S\left(x_{i}\right)$ and the root of a new arrival gadget $A\left(x_{i}, C_{j}\right)$ with odd length

$$
\ell_{x_{i}, C_{j}}=k-\left(2+\ell_{C_{j}}+\ell_{c}+1+\ell_{x_{i}, \overline{x_{i}}}+2+\ell_{x_{i}}^{\prime}\right)=k-\left(5+\ell_{C_{j}}+\left\lfloor\frac{8}{10} k\right\rfloor+\ell_{x_{i}, \overline{x_{i}}}\right) .
$$

Note that $\ell_{x_{i}, C_{j}}<\left\lfloor\frac{2}{10} k\right\rfloor$. We repeat the exact same operation with $\overline{x_{i}}$ and the clauses containing $\overline{x_{i}}$, resulting in gadgets $S\left(\overline{x_{i}}\right)$ with length $\ell_{\overline{x_{i}}}^{\prime}=\left\lfloor\frac{6}{10} k\right\rfloor$ and $A\left(\overline{x_{i}}, C_{j}\right)$ with odd length

$$
\ell_{\overline{x_{i}}, C_{j}}=k-\left(2+\ell_{C_{j}}+\ell_{c}+1+\ell_{x_{i}, \overline{x_{i}}}+2+\ell_{\overline{x_{i}}}^{\prime}\right)=k-\left(5+\ell_{C_{j}}+\left\lfloor\frac{8}{10} k\right\rfloor+\ell_{x_{i}, \overline{x_{i}}}\right),
$$

smaller than $\left\lfloor\frac{2}{10} k\right\rfloor$. Note that the construction of $T$ and $M$ is achieved in polynomial time.

Prior to explain why the reduction is correct, let us point out a few facts. The exposed nodes of $T$ are the following:

- the forth- and back-nodes of all departure gadgets $G\left(x_{i}\right)$;

- the forth- and back-nodes of all departure gadgets $G\left(C_{j}\right)$;

- the in- and out-nodes of all gate gadgets $S\left(x_{i}\right)$ and $S\left(\overline{x_{i}}\right)$;

- the in- and out-nodes of all arrival gadgets $A\left(x_{i}, C_{j}\right)$ and $A\left(\overline{x_{i}}, C_{j}\right)$.

Among all pairs of exposed nodes, due to our choice of some the gadgets' and paths' lengths, note that only the following ones are at distance exactly $k$ in $T$ (see Figure 6 for an illustration):

1. For every variable $x_{i}$ of $\Phi$, the forth-node of $G\left(x_{i}\right)$ and the in-nodes of $S\left(x_{i}\right)$ and $S\left(\overline{x_{i}}\right)$. 


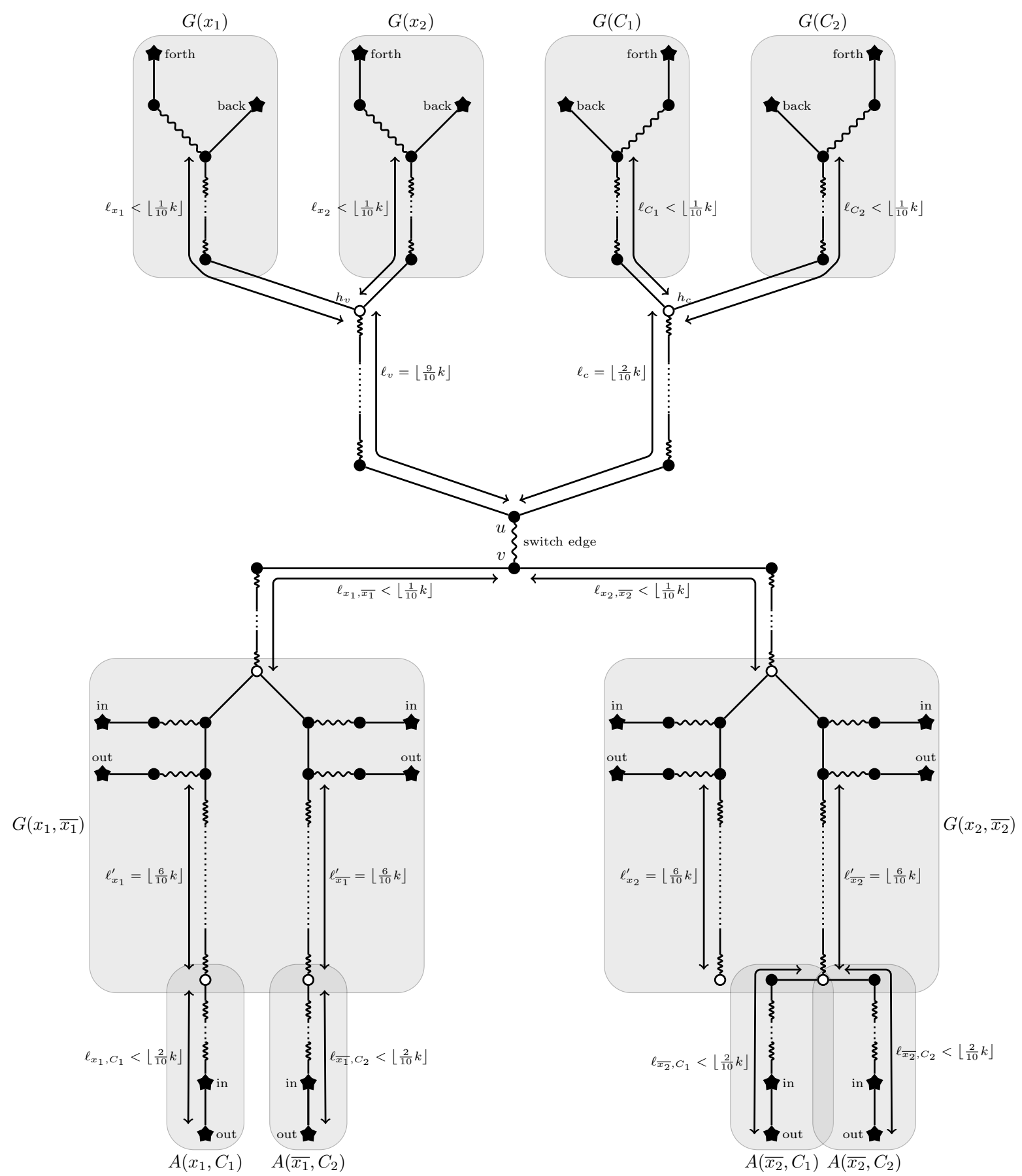

Figure 6: Illustration of the reduction described in the proof of Theorem 3.5, for a formula $\Phi$ having two variables $x_{1}$ and $x_{2}$ and two clauses $C_{1}$ and $C_{2}$ such that $x_{1}, \overline{x_{2}} \in C_{1}$ and $\overline{x_{1}}, \overline{x_{2}} \in C_{2}$. Star nodes are exposed nodes. Wiggly edges are edges of the matching.

2. For every variable $x_{i}$ of $\Phi$, the back-node of $G\left(x_{i}\right)$ and the out-nodes of $S\left(x_{i}\right)$ and $S\left(\overline{x_{i}}\right)$.

3. For every clause $C_{j}$ and variable $x_{i} \in C_{j}$ (or negated variable $\overline{x_{i}} \in C_{j}$ ) in $\Phi$, the forth-node of $G\left(C_{j}\right)$ and the in-node of $A\left(x_{i}, C_{j}\right)$ (resp. $A\left(\overline{x_{i}}, C_{j}\right)$ ).

4. for every clause $C_{j}$ and variable $x_{i} \in C_{j}$ (or negated variable $\overline{x_{i}} \in C_{j}$ ) in $\Phi$, the back-node of $G\left(C_{j}\right)$ and the out-node of $A\left(x_{i}, C_{j}\right)$ (resp. $A\left(\overline{x_{i}}, C_{j}\right)$ ).

We now explain how $M$ should be $(=k)$-augmented to a matching of size $\mu_{=k}(T, M)$. First 
of all, it should be noted that, at any point, if a $(=k)$-augmentation is performed from the forth-node of a departure gadget $G_{1}$ to the in-node of a gate or arrival gadget $G_{2}$, then only the $(=k)$-augmentation from the back-node of $G_{1}$ to the out-node of $T$ can be performed. This is because the node $u$ (the end of the switch edge that is the closest to the departure gadgets) is now covered by an incident edge on the path between $u$ and $G_{1}$. So, in a sense, $(=k)$-augmentations have to be performed in pairs. Assuming the two $(=k)$-augmentations from $G\left(x_{i}\right)$ to $S\left(x_{i}\right)$ (resp., to $S\left(\overline{x_{i}}\right)$ ) have been performed, we say that the gate gadget $S\left(x_{i}\right)$ (resp. $S\left(\overline{x_{i}}\right)$ ) is open.

As exposed above, the possible $(=k)$-augmentations go from a departure gadget $G\left(x_{i}\right)$ or $G\left(C_{j}\right)$ towards either a gate gadget (case where the departure gadget is a $G\left(x_{i}\right)$ ), or an arrival gadget (otherwise). Assuming the clause $C_{j}$ of $\Phi$ contains the variable $x_{i}$ (resp. negated variable $\overline{x_{i}}$ ), we note that augmenting a $(=k)$-path from $G\left(C_{j}\right)$ to $A\left(x_{i}, C_{j}\right)$ (resp. $A\left(\overline{x_{i}}, C_{j}\right)$ cannot be done until $S\left(x_{i}\right)$ (resp. $S\left(\overline{x_{i}}\right)$ ) is open. Furthermore, we note that once a gate, say $S\left(x_{i}\right)$, is open, assuming $x_{i}$ is contained is distinct clauses $C_{j_{1}}, \cdots, C_{j_{n_{i}}}$ of $\Phi$, all pairs of $(=k)$-augmentations from the departure gadgets $G\left(C_{j_{1}}\right), \cdots, G\left(C_{j_{n_{i}}}\right)$ to the arrival gadgets $A\left(x_{i}, C_{j_{1}}\right), \cdots, A\left(x_{i}, C_{j_{n_{i}}}\right)$ can be performed in turns.

To sum up, a matching of size $\mu_{=k}(T, M)$ can be obtained, via $(=k)$-augmentations starting from $M$, as follows:

1. Consider all of the variables $x_{1}, \cdots, x_{n}$ of $\Phi$ in turn, and, for every considered variable $x_{i}$, either perform the two augmentations from $G\left(x_{i}\right)$ to $S\left(x_{i}\right)$ (i.e., open $S\left(x_{i}\right)$ ), or perform the two augmentations from $G\left(x_{i}\right)$ to $S\left(\overline{x_{i}}\right)$ (i.e., open $S\left(\overline{x_{i}}\right)$ ),

2. Then consider all of the clauses $C_{1}, \cdots, C_{m}$ of $\Phi$ in turn, and, for every considered clause $C_{j}$, if $C_{j}$ contains one variable $x_{i}$ (resp. negated variable $\left.\overline{x_{i}}\right)$ such that $S\left(x_{i}\right)\left(\operatorname{resp} . S\left(\overline{x_{i}}\right)\right)$ is open, then perform the two augmentations from $G\left(C_{j}\right)$ to $A\left(x_{i}, C_{j}\right)\left(\operatorname{resp} . A\left(\overline{x_{i}}, C_{j}\right)\right)$.

So $2 n+|M| \leq \mu_{=k}(T, M) \leq 2 n+2 m+|M|$, and the upper bound is attained when, for every variable $x_{i}$, we can open either $S\left(x_{i}\right)$ or $S\left(\overline{x_{i}}\right)$ so that, for every clause gadget $C_{j}$, there is an open gate gadget creating a way from the departure gadget $G\left(C_{j}\right)$ towards an arrival gadget $A\left(x_{i}, C_{j}\right)$ or $A\left(\overline{x_{i}}, C_{j}\right)$. By considering that opening $S\left(x_{i}\right)$ (resp. $S\left(\overline{x_{i}}\right)$ ) simulates the affectation of value true (resp. false) to $x_{i}$, and that performing a pair of $(=k)$-augmentations from $G\left(C_{j}\right)$ to $A\left(x_{i}, C_{j}\right)$ (or $A\left(\overline{x_{i}}, C_{j}\right)$ ) simulates the fact that $x_{i}$ (resp. $\overline{x_{i}}$ ) brings value true to $C_{j}$, the equivalence with satisfying $\Phi$ follows.

\section{Conclusion}

Following the work of Nisse, Salch and Weber [NSW15], we have, in this paper, studied the algorithmic complexity of $M P^{\leq k}$, the problem of augmenting an initial matching as much as possible via $(\leq k)$-augmentations. As this case is far from being well understood, we gave a special focus to the case of trees. On the positive side, we have provided polynomial-time algorithms for solving the problem in bounded-degree trees (assuming $k$ is also fixed), $k$-sparse trees, and caterpillars.

Seeking for the complexity of $M P^{\leq k}$ in trees, we have introduced a more restricted version of the problem, $M P^{=k}$. On the negative side, we have proved that this problem is indeed NP-complete in trees, when $k$ is part of the input.

One important point to raise, is that the polynomial-time algorithms we have proposed for $M P^{\leq k}$ also apply for $M P^{=k}$. To see this is true, one should keep in mind that our algorithms heavily rely on the point that, when given a sequence of augmentations, we can disentangle the augmented paths (i.e., make them disjoint) and get an equivalent sequence. When considering the $M P^{=k}$ problem, such a property is not needed, as it can easily be checked that, for any two augmenting paths with the same length, in a caterpillar or a $k$-sparse tree one of the two paths cannot completely include the other.

In the rest of this section, we summarize a number of directions for further work on this topic. 


\section{Complexity of $M P \leq k$}

Our perspectives for future work are mainly about the complexity of $M P^{\leq k}$ in more classes of trees, towards a full understanding of the problem in that class of graphs. In the current paper, so that the arguments in our proofs work, we needed strong assumptions on the tree's structure, such as long distances between the b-nodes ( $k$-sparse trees), or short branches attached to the b-nodes (caterpillars). The next step would hence be to consider classes of trees without those properties.

Our main interest for focusing on caterpillars in this paper, is that their structure is close to that of paths, for which it exists a polynomial-time solving algorithm for $M P^{\leq k}$. We have the feeling that, from an algorithm solving $M P^{\leq k}$ for a tree $T$, one could deduce one for any subdivision of $T$, as the subdivision operation introduces new degree-2 nodes only. Hence, we believe the following could be considered:

Question 4.1. What is the complexity of $M P^{\leq k}$ when restricted to subdivided caterpillars?

Remind that, in the case of caterpillars, a key fact is that the maximum degree can be assumed to be at most 3 (due to Corollary 2.19). Obviously, this does not remain true in subdivided caterpillars. Towards Question 4.1, a simpler generalization of our result for caterpillars could hence be to consider combs, i.e., subdivided caterpillars with maximum degree 3 . In particular, a quick investigation shows that, already in that class of trees, performing augmentations from left to right might not be an optimal strategy.

Question 4.2. What is the complexity of $M P^{\leq k}$ when restricted to combs?

Our result on $k$-sparse trees stands as a generalization of arguments for solving $M P^{\leq k}$ in subdivided stars. In that light, perhaps another class of trees that could be interesting considering is the one of subdivided bistars (i.e., trees with exactly two b-nodes). Note that, in a subdivided bistar $S$, if the two b-nodes are at distance more than $k$, then $S$ is a $k$-sparse tree, in which case we know how to solve $M P^{\leq k}$. Hence, in order to understand how $(\leq k)$-augmentations should be performed through b-nodes, the following case could be considered:

Question 4.3. What is the complexity of $M P^{\leq k}$ when restricted to bistars?

Complexity of $M P^{=k}$

We believe directions concerning $M P^{=k}$ are also worth considering. In particular, although we proved that $M P^{=}$is NP-complete in trees, we have no clue about whether this is true for $M P^{=k}$, for some fixed $k$.

Question 4.4. Is there an odd $k \geq 3$ such that $M P^{=k}$ is NP-complete when restricted to trees?

In particular, the case $k=3$ sounds intriguing:

Question 4.5. What is the complexity of $M P^{=3}$ when restricted to trees?

\section{Other classes of graphs}

Although $M P^{\leq k}$ and $M P^{=k}$ remain NP-complete when restricted to rather restricted classes of graphs (with maximum degree 3, degeneracy 2, arbitrarily large girth, planarity, bounded treewidth), the polynomial-time solvable cases should concern particular classes of graphs with convenient properties. As such classes, one could consider $\Theta$-graphs (graphs with two vertices joined by arbitrarily many disjoint paths with arbitrary lengths), cacti (graphs made up of cycles connected in a tree-like fashion), or interval graphs. The latter class sounds rather interesting, as perhaps, in that case, augmentations should be performed from left to right, just as in the path and caterpillar cases. 


\section{References}

[Ber57] C. Berge. Two theorems in graph theory. Proceedings of the National Academy of Sciences of the United States of America, 43(9):842-844, September 1957.

[BSS09] L. Bui, S. Sanghavi, and R. Srikant. Distributed link scheduling with constant overhead. IEEE/ACM Trans. Netw., 17(5):1467-1480, 2009.

[DP14] R. Duan and S. Pettie. Linear-time approximation for maximum weight matching. $J$. ACM, 61(1), 2014.

[Edm65] J. Edmonds. Paths, trees, and flowers. Canadian Journal of Mathematics, 17:449-467, 1965.

[HK73] J. E. Hopcroft and R. M. Karp. An $n^{5 / 2}$ algorithm for maximum matchings in bipartite graphs. SIAM J. Comput., 2(4):225-231, 1973.

[Kuh55] H. W. Kuhn. The hungarian method for the assignment problem. Naval Research Logistics Quarterly, 2:83-97, 1955.

[MV80] S. Micali and V. V. Vazirani. An $O(\sqrt{|V|}|E|)$ algorithm for finding maximum matching in general graphs. In 21st Symp. on Foundations of Comp. Sc. (FOCS), pages 17-27. IEEE, 1980.

[NSW15] N. Nisse, A. Salch, and V. Weber. Recovery of disrupted airline operations, 2015. INRIARR-8679, http://hal.inria.fr/Something.

[WS05] X. Wu and R. Srikant. Regulated maximal matching: A distributed scheduling algorithm for multi-hop wireless networks with nodeexclusive spectrum sharing. In IEEE Conf. on Decision and Control, 2005. 